\title{
A study of quasi-elastic muon neutrino and antineutrino scattering in the NOMAD experiment
}

V. Lyubushkin ${ }^{6, a}$, B. Popov ${ }^{6,14}$, J.J. Kim ${ }^{19}$, L. Camilleri ${ }^{8}$, J.-M. Levy ${ }^{14}$, M. Mezzetto ${ }^{13}$, D. Naumov ${ }^{6,7}$, S. Alekhin ${ }^{25}$, P. Astier ${ }^{14}$, D. Autiero ${ }^{8}$, A. Baldisseri ${ }^{18}$, M. Baldo-Ceolin ${ }^{13}$, M. Banner ${ }^{14}$, G. Bassompierre ${ }^{1}$, K. Benslama ${ }^{9}$, N. Besson ${ }^{18}$, I. Bird ${ }^{8,9}$, B. Blumenfeld ${ }^{2}$, F. Bobisut ${ }^{13}$, J. Bouchez ${ }^{18}$, S. Boyd ${ }^{20}$, A. Bueno ${ }^{3,24}$, S. Bunyatov ${ }^{6}$, A. Cardini ${ }^{10}$, P.W. Cattaneo ${ }^{15}$, V. Cavasinni ${ }^{16}$, A. Cervera-Villanueva ${ }^{8,22}$, R. Challis ${ }^{11}$, A. Chukanov ${ }^{6}$, G. Collazuol $^{13}$, G. Conforto ${ }^{8,21, b}$, C. Conta ${ }^{15}$, M. Contalbrigo ${ }^{13}$, R. Cousins ${ }^{10}$, D. Daniels ${ }^{3}$, H. Degaudenzi ${ }^{9}$, T. Del Prete ${ }^{16}$, A. De Santo ${ }^{8,16}$, T. Dignan ${ }^{3}$, L. Di Lella ${ }^{8, c}$, E. do Couto e Silva ${ }^{8}$, J. Dumarchez $^{14}$, M. Ellis ${ }^{20}$, G.J. Feldman ${ }^{3}$, R. Ferrari ${ }^{15}$, D. Ferrère ${ }^{8}$, V. Flaminio ${ }^{16}$, M. Fraternali ${ }^{15}$, J.-M. Gaillard ${ }^{1}$, E. Gangler ${ }^{8,14}$, A. Geiser ${ }^{5,8}$, D. Geppert ${ }^{5}$, D. Gibin ${ }^{13}$, S. Gninenko ${ }^{8,12}$, A. Godley ${ }^{19}$, J.-J. Gomez-Cadenas ${ }^{8,22}$, J. Gosset ${ }^{18}$,

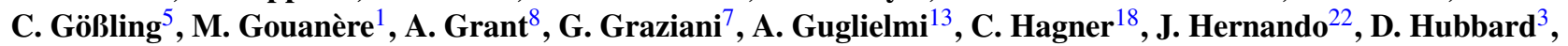
P. Hurst ${ }^{3}$, N. Hyett ${ }^{11}$, E. Iacopini ${ }^{7}$, C. Joseph ${ }^{9}$, F. Juget ${ }^{9}$, N. Kent ${ }^{11}$, M. Kirsanov ${ }^{12}$, O. Klimov ${ }^{6}$, J. Kokkonen ${ }^{8}$,

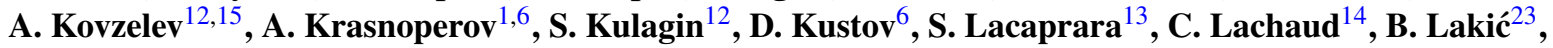
A. Lanza ${ }^{15}$, L. La Rotonda ${ }^{4}$, M. Laveder ${ }^{13}$, A. Letessier-Selvon ${ }^{14}$, J. Ling ${ }^{19}$, L. Linssen ${ }^{8}$, A. Ljubičić ${ }^{23}$, J. Long ${ }^{2}$, A. Lupi ${ }^{7}$, A. Marchionni ${ }^{7}$, F. Martelli ${ }^{21}$, X. Méchain ${ }^{18}$, J.-P. Mendiburu ${ }^{1}$, J.-P. Meyer ${ }^{18}$, S.R. Mishra ${ }^{19}$, G.F. Moorhead ${ }^{11}$, P. Nédélec ${ }^{1}$, Yu. Nefedov ${ }^{6}$, C. Nguyen-Mau $^{9}$, D. Orestano ${ }^{17}$, F. Pastore ${ }^{17}$, L.S. Peak ${ }^{20}$, E. Pennacchio ${ }^{21}$, H. Pessard ${ }^{1}$, R. Petti ${ }^{19}$, A. Placci ${ }^{8}$, G. Polesello ${ }^{15}$, D. Pollmann ${ }^{5}$, A. Polyarush ${ }^{12}$, C. Poulsen ${ }^{11}$, L. Rebuffi ${ }^{13}$, J. Rico ${ }^{24}$, P. Riemann ${ }^{5}$, C. Roda ${ }^{8,16}$, A. Rubbia ${ }^{8,24}$, F. Salvatore ${ }^{15}$, O. Samoylov ${ }^{6}$, K. Schahmaneche ${ }^{14}$, B. Schmidt ${ }^{5,8}$, T. Schmidt ${ }^{5}$, A. Sconza ${ }^{13}$, M. Seaton ${ }^{19}$, M. Sevior ${ }^{11}$, D. Sillou ${ }^{1}$, F.J.P. Soler ${ }^{8,20}$, G. Sozzi ${ }^{9}$, D. Steele ${ }^{2,9}$, U. Stiegler ${ }^{8}$, M. Stipčević ${ }^{23}$, Th. Stolarczyk ${ }^{18}$, M. Tareb-Reyes ${ }^{9}$, G.N. Taylor ${ }^{11}$, V. Tereshchenko ${ }^{6}$, A. Toropin $^{12}$, A.-M. Touchard ${ }^{14}$, S.N. Tovey ${ }^{8,11}$, M.-T. Tran ${ }^{9}$, E. Tsesmelis ${ }^{8}$, J. Ulrichs ${ }^{20}$, L. Vacavant ${ }^{9}$, M. Valdata-Nappi ${ }^{4, \mathrm{~d}}$, V. Valuev ${ }^{6,10}$, F. Vannucci ${ }^{14}$, K.E. Varvell ${ }^{20}$, M. Veltri ${ }^{21}$, V. Vercesi ${ }^{15}$, G. Vidal-Sitjes ${ }^{8}$, J.-M. Vieira ${ }^{9}$, T. Vinogradova $^{10}$, F.V. Weber ${ }^{3,8}$, T. Weisse ${ }^{5}$, F.F. Wilson ${ }^{8}$, L.J. Winton ${ }^{11}$, Q. Wu ${ }^{19}$, B.D. Yabsley ${ }^{20}$, H. Zaccone ${ }^{18}$, K. Zuber ${ }^{5}$, P. Zuccon ${ }^{13}$

${ }^{1}$ LAPP, Annecy, France

${ }^{2}$ Johns Hopkins Univ., Baltimore, MD, USA

${ }^{3}$ Harvard Univ., Cambridge, MA, USA

${ }^{4}$ Univ. of Calabria and INFN, Cosenza, Italy

${ }^{5}$ Dortmund Univ., Dortmund, Germany

${ }^{6}$ JINR, Dubna, Russia

${ }^{7}$ Univ. of Florence and INFN, Florence, Italy

${ }^{8}$ CERN, Geneva, Switzerland

${ }^{9}$ University of Lausanne, Lausanne, Switzerland

${ }^{10}$ UCLA, Los Angeles, CA, USA

${ }^{11}$ University of Melbourne, Melbourne, Australia

${ }^{12}$ Inst. for Nuclear Research, INR, Moscow, Russia

${ }^{13}$ Univ. of Padova and INFN, Padova, Italy

${ }^{14}$ LPNHE, Univ. of Paris VI and VII, Paris, France

${ }^{15}$ Univ. of Pavia and INFN, Pavia, Italy

${ }^{16}$ Univ. of Pisa and INFN, Pisa, Italy

${ }^{17}$ Roma Tre University and INFN, Rome, Italy

${ }^{18}$ DAPNIA, CEA, Saclay, France

${ }^{19}$ Univ. of South Carolina, Columbia, SC, USA

${ }^{20}$ Univ. of Sydney, Sydney, Australia

${ }^{21}$ Univ. of Urbino, Urbino, and INFN, Florence, Italy

${ }^{22}$ IFIC, Valencia, Spain

${ }^{23}$ Rudjer Bošković Institute, Zagreb, Croatia

${ }^{24}$ ETH Zùrich, Zùrich, Switzerland

${ }^{25}$ Inst. for High Energy Physics, 142281, Protvino, Moscow Region, Russia 
Received: 19 January 2009 / Revised: 17 July 2009 / Published online: 21 August 2009

(C) Springer-Verlag / Società Italiana di Fisica 2009

Abstract We have studied the muon neutrino and antineutrino quasi-elastic (QEL) scattering reactions $\left(v_{\mu} n \rightarrow \mu^{-} p\right.$ and $\bar{v}_{\mu} p \rightarrow \mu^{+} n$ ) using a set of experimental data collected by the NOMAD Collaboration. We have performed measurements of the cross-section of these processes on a nuclear target (mainly carbon) normalizing it to the total $v_{\mu}\left(\bar{v}_{\mu}\right)$ charged-current cross section. The results for the flux-averaged QEL cross sections in the (anti)neutrino energy interval $3-100 \mathrm{GeV}$ are $\left\langle\sigma_{\mathrm{qel}}\right\rangle_{v_{\mu}}=(0.92 \pm 0.02$ (stat) \pm 0.06 (syst) $) \times 10^{-38} \mathrm{~cm}^{2}$ and $\left\langle\sigma_{\mathrm{qel}}\right\rangle_{\bar{\nu}_{\mu}}=(0.81 \pm 0.05$ (stat) \pm 0.09 (syst) $) \times 10^{-38} \mathrm{~cm}^{2}$ for neutrino and antineutrino, respectively. The axial mass parameter $M_{A}$ was extracted from the measured quasi-elastic neutrino cross section. The corresponding result is $M_{A}=1.05 \pm 0.02$ (stat) \pm 0.06 (syst) GeV. It is consistent with the axial mass values recalculated from the antineutrino cross section and extracted from the pure $Q^{2}$ shape analysis of the high purity sample of $v_{\mu}$ quasielastic 2-track events, but has smaller systematic error and should be quoted as the main result of this work. Our measured $M_{A}$ is found to be in good agreement with the world average value obtained in previous deuterium filled bubble chamber experiments. The NOMAD measurement of $M_{A}$ is lower than those recently published by $\mathrm{K} 2 \mathrm{~K}$ and MiniBooNE Collaborations. However, within the large errors quoted by these experiments on $M_{A}$, these results are compatible with the more precise NOMAD value.

PACS $13.15 .+\mathrm{g} \cdot 25.30 . \mathrm{Pt}$

\section{Introduction}

A precise knowledge of the cross section of (anti)neutrinonucleus quasi-elastic scattering process (QEL) is important for the planning and analysis of any experiment which detects astrophysical, atmospheric or accelerator neutrinos. The available measurements from early experiments at ANL [1-4], BNL [5-8], FNAL [9, 10], CERN [11-18] and IHEP [19-22] have considerable errors due to low statistics and a lack of knowledge of the precise incoming neutrino flux. Unfortunately, even within these large errors, the results are often conflicting.

This subject remains very topical. Recently several attempts have been made to investigate the QEL process

\footnotetext{
a e-mail: Vladimir.Lyubushkin@ cern.ch

${ }^{\mathrm{b}}$ Deceased

${ }^{\mathrm{c}}$ Now at Scuola Normale Superiore, Pisa, Italy

${ }^{\mathrm{d}}$ Now at Univ. of Perugia and INFN, Perugia, Italy
}

in the data collected by modern accelerator neutrino experiments (such as NuTeV [23], K2K [24, 25] and MiniBooNE [26]). Unfortunately they have not clarified the situation again due to large errors assigned to their measurements. Dedicated experiments, such as e.g. SciBooNE [27] and MINER $v$ A [28], are now being performed.

In the present analysis, we study both $v_{\mu}$ and $\bar{v}_{\mu}$ QEL scattering in the data collected by the NOMAD Collaboration. The NOMAD detector was exposed to a wide-band neutrino beam produced by the $450 \mathrm{GeV}$ proton synchrotron (SPS, CERN). A detailed description of the experimental set-up can be found in [29]. The characteristics of the incoming neutrino flux are given in [30].

The large amount of collected data and the good quality of event reconstruction in the NOMAD detector provide a unique possibility to measure the QEL cross section with a combination of small statistical and systematic errors. The data sample used in this analysis consists of about $751000(23000) v_{\mu}\left(\bar{v}_{\mu}\right)$ charged-current (CC) interactions in a reduced detector fiducial volume. The average energy of the incoming $v_{\mu}\left(\bar{v}_{\mu}\right)$ is 25.9 (17.6) GeV.

The merit of the current analysis is the possibility of keeping the systematic error relatively small. It takes advantage from three main factors: (1) the NOMAD detector is capable of selecting a sample of QEL events with a high purity and a good efficiency; (2) a simultaneous measurement of both two track and single track $v_{\mu}$ QEL events allows one to constrain the systematics associated with nuclear reinteractions; (3) a wide energy range of the NOMAD neutrino beam allows one to perform a precise normalization to the well-known total (DIS) $v_{\mu}$ CC cross section.

The paper is organized as follows. In Sect. 2 we give a brief review of the published experimental data on QEL (anti)neutrino scattering. The NOMAD detector and the incoming neutrino flux are briefly discussed in Sect. 3. In Sect. 4 we outline the MC modeling of signal and background events, emphasizing also the importance of nuclear effects. Section 5 is devoted to the selection of the QEL events; we describe the QEL identification procedure and compare the MC predictions with experimental data. The methods used to measure the QEL cross section and the phenomenological axial mass parameter $M_{A}$ are the subjects of Sect. 6. The systematic uncertainties are summarized in Sect. 7. The results are presented in Sect. 8. Finally, a summary and discussion of the obtained results are given in Sect. 9.

More detailed information can be found in [31]. 


\section{Review of existing experimental data on quasi-elastic (anti)neutrino scattering}

Let us start with a brief review of existing experimental data on (anti)neutrino-nucleon QEL scattering.

A compilation of available data on the cross section measurement of the $v_{\mu}$ and $\bar{v}_{\mu}$ quasi-elastic scattering off deuterium and other nuclei or composite targets (like freon, propane, liquid scintillator) as a function of the incoming neutrino energy has been made (see Figs. 17, 18 and 19). This study allowed one to conclude that the QEL cross section measured in different experiments can vary by $20-40 \%$.

The existing data on (anti)neutrino QEL scattering come mostly from bubble chamber (BC) experiments. In general, these data suffer from small statistics. Moreover, results of several old experiments [12-14] have large systematic uncertainties due to the poor knowledge of the incoming neutrino flux and of background contamination in the selected events.

The total QEL cross section was recently measured in data collected by the NuTeV Collaboration [23]. The number of QEL events identified in their analysis are comparable with the total world data obtained in previous experiments. However, the results reported for the antineutrino case fall well outside the most probable range of values known today and hence, seem to exhibit a systematic shift.

Another intriguing subject in the study of the neutrino quasi-elastic scattering is the axial structure of the nucleon. We will skip here the details of the phenomenology of the hadronic current involved in the matrix element of the process (see Sect. 4.1 and [32]). But let us only remind the reader that for the region of low and intermediate 4-momentum transfer, $Q^{2}$, we can use a dipole parameterization for the axial form factor with only one adjustable parameter, the so-called axial mass $M_{A}$.

The $M_{A}$ parameter describes the internal structure of the nucleon and should be the same both for neutrino and antineutrino experiments (if we assume the isotopic invariance of strong interaction). Therefore, it is convenient to compare experimental results in terms of the axial mass. There is, however, no theoretical basis for this form of the axial form factor. The use of an inappropriate parameterization could therefore lead to values of $M_{A}$ that differ when extracted under different kinematical conditions.

There are two possible ways generally used to extract the $M_{A}$ parameter from experimental data:

1. from the total QEL (anti)neutrino-nucleon cross section (the axial form factor is responsible for about $50-60 \%$ of the total QEL cross section);

2. from the fit of the $Q^{2}$ distribution of the identified neutrino QEL events.

In principle, these two procedures should give selfconsistent results. However, the old bubble chamber exper- iments at ANL and CERN reported in general larger values of $M_{A}$ based on the $Q^{2}$ fit than those obtained from the total cross-section measurements.

Results of the $M_{A}$ measurements based on the $Q^{2}$ fit have been recently published by the K2K $[24,25]$ and MiniBooNE [26] Collaborations. They are about $15 \%$ higher than the average of previous deuterium filled bubble chamber experiments. This disagreement is, however, just at about one sigma level because of the large systematic errors associated with the K2K and MiniBooNE measurements.

Let us note that the extraction of $M_{A}$ from the $Q^{2}$ distribution fit is a more delicate issue than the QEL total cross section measurement.

In general, there are at least three aspects which can affect noticeably the $M_{A}$ measurements:

1. The nuclear effects can distort the expected distributions of the measured kinematic variables (like the energy of the outgoing nucleon). The neutrino-nucleus interactions should be described by a theoretical model suitable for the considered neutrino energy region. This is important both for MC modeling in present-day neutrino experiments and for a proper interpretation of the results obtained earlier (with few exceptions for the deuterium filled bubble chambers).

2. The correct determination of the background contamination from both deep inelastic scattering and single-pion production in the selected events is important for experiments operating with intermediate and high energy neutrino beams.

3. The QEL reconstruction efficiency as a function of $Q^{2}$ for two-track events is not expected to be a flat function. It should drop both at small $Q^{2}$ due to the loss of low energy protons and at large $Q^{2}$ due to the loss of low energy muons. Effects which influence the efficiency of the low momentum particle reconstruction should be carefully taken into account in the MC modeling of the detector response.

Tables 1 and 2 display the measured values of $M_{A}$ from neutrino and antineutrino experiments (this compilation is also presented in graphical form in Fig. 20). Whenever possible we provide also the $M_{A}$ measured from the total cross section.

From the results described above one can conclude that the presently available experimental data on the neutrino QEL cross section allow for a very wide spread of the axial mass values, roughly from 0.7 to $1.3 \mathrm{GeV}$. Therefore the reliability of a theoretical fit to these data is questionable and the uncertainty attributed to such a fit should go beyond the averaged experimental statistical accuracy. Nevertheless, the formal averaging of $M_{A}$ values from several early experiments was done by the authors of [33]: $M_{A}=$ $1.026 \pm 0.021 \mathrm{GeV}$. This result is also known as the axial 
Table 1 A summary of existing experimental data: the axial mass $M_{A}$ as measured in previous neutrino experiments. Numbers of observed events have been taken from the original papers; usually they are not corrected for efficiency and purity (the so-called QEL candidates). The axial mass value for the $\mathrm{NuTeV}$ experiment [23] was estimated from the published neutrino quasi-elastic cross section $\left(\sigma_{v}^{\text {qel }}=(0.94 \pm 0.03\right.$ (stat $) \pm$ 0.07 (syst) $) \times 10^{-38} \mathrm{~cm}^{2}$ ); the systematic error for IHEP SKAT 90 [22] is $0.14 \mathrm{GeV}$

\begin{tabular}{|c|c|c|c|c|c|}
\hline Experiment & Target & Events & Method & $M_{A}, \mathrm{GeV}$ & Ref. \\
\hline \multirow[t]{2}{*}{ ANL 69} & \multirow[t]{2}{*}{ Steel } & & $d \sigma / d Q^{2}$ & $1.05 \pm 0.20$ & [1] \\
\hline & & & $\sigma$ & $0.97 \pm 0.16$ & \\
\hline \multirow[t]{3}{*}{ ANL 73} & \multirow[t]{3}{*}{ Deuterium } & \multirow[t]{3}{*}{166} & $d \sigma / d Q^{2}$ & $0.94 \pm 0.18$ & \multirow[t]{3}{*}[2]{} \\
\hline & & & $\sigma \otimes d \sigma / d Q^{2}$ & $0.95 \pm 0.12$ & \\
\hline & & & $\sigma$ & $0.75_{-0.11}^{+0.13}$ & \\
\hline \multirow[t]{3}{*}{ ANL 77} & \multirow[t]{3}{*}{ Deuterium } & \multirow[t]{3}{*}{$\sim 600$} & $d \sigma / d Q^{2}$ & $1.01 \pm 0.09$ & \multirow[t]{3}{*}[3]{} \\
\hline & & & $\sigma \otimes d \sigma / d Q^{2}$ & $0.95 \pm 0.09$ & \\
\hline & & & $\sigma$ & $0.74 \pm 0.12$ & \\
\hline \multirow[t]{2}{*}{ ANL 82} & \multirow[t]{2}{*}{ Deuterium } & \multirow[t]{2}{*}{1737} & $d \sigma / d Q^{2}$ & $1.05 \pm 0.05$ & \multirow[t]{2}{*}[4]{} \\
\hline & & & $\sigma \otimes d \sigma / d Q^{2}$ & $1.03 \pm 0.05$ & \\
\hline BNL 81 & Deuterium & 1138 & $d \sigma / d Q^{2}$ & $1.07 \pm 0.06$ & {$[6]$} \\
\hline BNL 90 & Deuterium & 2538 & $d \sigma / d Q^{2}$ & $1.070_{-0.045}^{+0.040}$ & {$[8]$} \\
\hline FermiLab 83 & Deuterium & 362 & $d \sigma / d Q^{2}$ & $1.05_{-0.16}^{+0.12}$ & [9] \\
\hline NuTeV 04 & Steel & 21614 & $\sigma$ & $1.11 \pm 0.08$ & [23] \\
\hline MiniBooNE 07 & Mineral oil & 193709 & $d \sigma / d Q^{2}$ & $1.23 \pm 0.20$ & [26] \\
\hline CERN HLBC 64 & Freon & 236 & $d \sigma / d Q^{2}$ & $1.00_{-0.20}^{+0.35}$ & [11] \\
\hline CERN HLBC 67 & Freon & 90 & $\sigma \otimes d \sigma / d Q^{2}$ & $0.75_{-0.20}^{+0.24}$ & {$[12]$} \\
\hline CERN SC 68 & Steel & 236 & $d \sigma / d Q^{2}$ & $0.65_{-0.40}^{+0.45}$ & {$[13]$} \\
\hline CERN HLBC 69 & Propane & 130 & $\sigma \otimes d \sigma / d Q^{2}$ & $0.70 \pm 0.20$ & [14] \\
\hline \multirow[t]{2}{*}{ CERN GGM 77} & \multirow[t]{2}{*}{ Freon } & \multirow[t]{2}{*}{687} & $\sigma$ & $0.88 \pm 0.19$ & \\
\hline & & & $d \sigma / d Q^{2}$ & $0.96 \pm 0.16$ & {$[15]$} \\
\hline \multirow[t]{2}{*}{ CERN GGM 79} & \multirow[t]{2}{*}{ Propane/Freon } & \multirow[t]{2}{*}{556} & $\sigma$ & $0.87 \pm 0.18$ & \\
\hline & & & $d \sigma / d Q^{2}$ & $0.99 \pm 0.12$ & [17] \\
\hline \multirow[t]{2}{*}{ CERN BEBC 90} & \multirow[t]{2}{*}{ Deuterium } & \multirow[t]{2}{*}{552} & $\sigma$ & $0.94 \pm 0.07$ & \\
\hline & & & $d \sigma / d Q^{2}$ & $1.08 \pm 0.08$ & [18] \\
\hline IHEP 82 & Aluminium & 898 & $d \sigma / d Q^{2}$ & $1.00 \pm 0.07$ & {$[19]$} \\
\hline IHEP 85 & Aluminium & 1753 & $d \sigma_{v+\bar{v}} / d Q^{2}$ & $1.00 \pm 0.04$ & [20] \\
\hline \multirow[t]{2}{*}{ IHEP SCAT 88} & \multirow[t]{2}{*}{ Freon } & \multirow[t]{4}{*}{464} & $\sigma \otimes d \sigma / d Q^{2}$ & $0.96 \pm 0.15$ & [21] \\
\hline & & & $\sigma$ & $1.08 \pm 0.07$ & \\
\hline \multirow[t]{2}{*}{ IHEP SCAT 90} & \multirow[t]{2}{*}{ Freon } & & $d \sigma / d Q^{2}$ & $1.05 \pm 0.07$ & \multirow[t]{2}{*}[22]{} \\
\hline & & & $\sigma \otimes d \sigma / d Q^{2}$ & $1.06 \pm 0.05$ & \\
\hline $\mathrm{K} 2 \mathrm{~K}$ 06, SciFi & Water & $\sim 12000$ & $d \sigma / d Q^{2}$ & $1.20 \pm 0.12$ & [24] \\
\hline K2K 08, SciBar & Carbon & & $d \sigma / d Q^{2}$ & $1.144 \pm 0.077$ & [25] \\
\hline
\end{tabular}

mass world average value. According to [34-36] an updated world average value from $v_{\mu}$-deuterium and pion electroproduction experiments is $M_{A}=1.014 \pm 0.014 \mathrm{GeV}$.

\section{The NOMAD detector}

The NOMAD detector [29] consisted of an active target of 44 drift chambers with a total fiducial mass of 2.7 tons, located in a 0.4 Tesla dipole magnetic field as shown in Fig. 1.
The $X \times Y \times Z$ total volume of the drift chambers is about $300 \times 300 \times 400 \mathrm{~cm}^{3}$.

Drift chambers [37], made of low $Z$ material served the dual role of a nearly isoscalar target ${ }^{1}$ for neutrino interactions and of tracking medium. The average density of the

${ }^{1}$ The NOMAD active target is nearly isoscalar $\left(n_{n}: n_{p}=47.56 \%\right.$ : $52.43 \%$ ) and consists mainly of carbon; a detailed description of the drift chamber composition can be found in [37]. 
Table 2 The same as in Table 1, but for antineutrino experiments. The axial mass value for the $\mathrm{NuTeV}$ experiment [23] was estimated from the published antineutrino quasi-elastic cross section $\left(\sigma_{\bar{v}}^{\text {qel }}=(1.12 \pm 0.04(\right.$ stat $) \pm$ 0.10 (syst) $\left.) \times 10^{-38} \mathrm{~cm}^{2}\right)$; the systematic error for IHEP SKAT 90 [22] is $0.20 \mathrm{GeV}$

\begin{tabular}{llclll}
\hline Experiment & Target & Events & Determined from & $M_{A}, \mathrm{GeV}$ & Ref. \\
\hline BNL 80 & Hydrogen & & $d \sigma / d Q^{2}$ & $0.9_{-0.3}^{+0.4}$ & {$[5]$} \\
BNL 88 & Liquid scint. & 2919 & $d \sigma / d Q^{2}$ & $1.09 \pm 0.04$ & {$[7]$} \\
\hline FermiLab 84 & Neon & 405 & $d \sigma / d Q^{2}$ & $0.99 \pm 0.11$ & {$[10]$} \\
NuTeV 04 & Steel & 15054 & $\sigma$ & $1.29 \pm 0.11$ & {$[23]$} \\
\hline CERN GGM 77 & Freon & 476 & $\sigma$ & $0.69 \pm 0.44$ & {$[15]$} \\
& & & $d \sigma / d Q^{2}$ & $0.94 \pm 0.17$ & \\
CERN GGM 79 & Propane/Freon & 766 & $\sigma$ & $0.84+0.08$ & {$[16]$} \\
& & & $d \sigma / d Q^{2}$ & $0.91 \pm 0.04$ & \\
\hline IHEP 85 & Aluminium & 854 & $d \sigma \sigma_{v+\bar{v}} / d Q^{2}$ & $1.00 \pm 0.04$ & {$[20]$} \\
IHEP SKAT 88 & Freon & 52 & $\sigma \otimes d \sigma / d Q^{2}$ & $0.72 \pm 0.23$ & {$[21]$} \\
& & & $\sigma$ & $0.62 \pm 0.16$ & \\
IHEP SKAT 90 & Freon & & $d \sigma / d Q^{2}$ & $0.79 \pm 0.11$ & {$[22]$} \\
& & & $\sigma \otimes d \sigma / d Q^{2}$ & $0.71 \pm 0.10$ & \\
\hline
\end{tabular}

Fig. 1 A side-view of the NOMAD detector

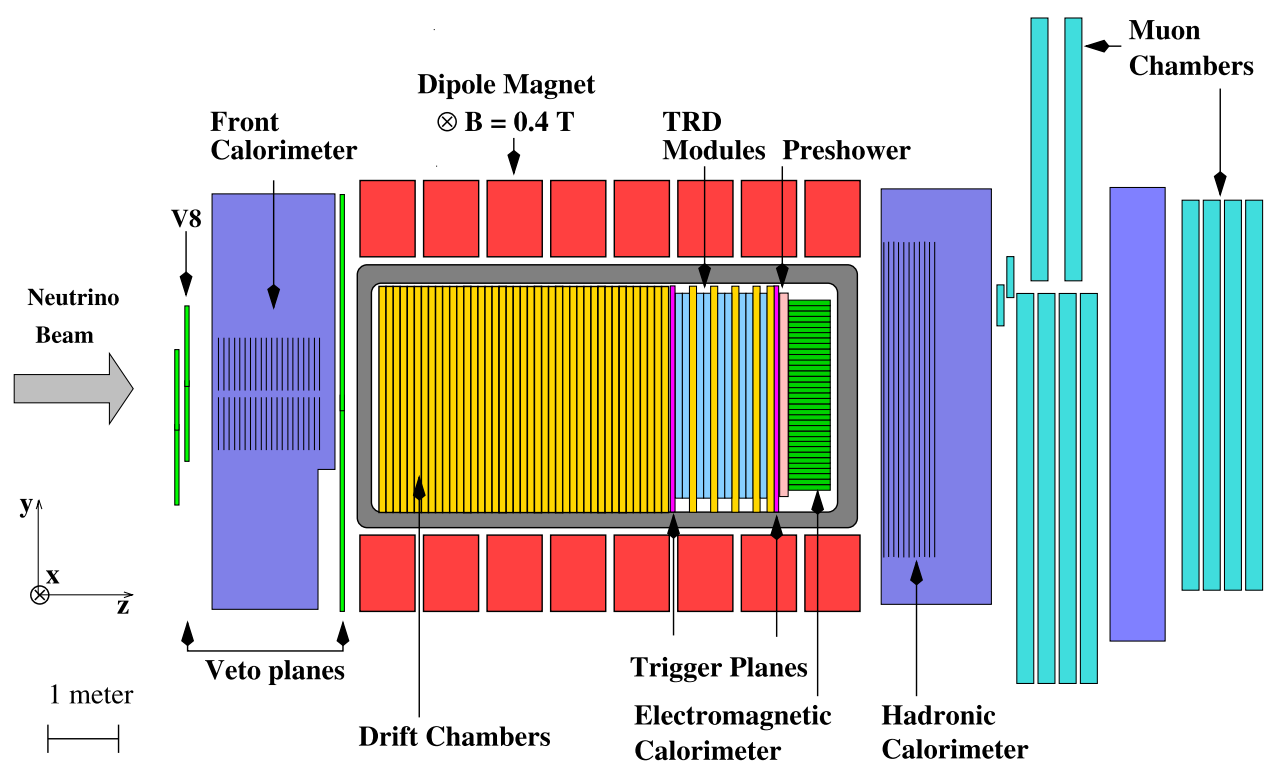

drift chamber volume was $0.1 \mathrm{~g} / \mathrm{cm}^{3}$. These chambers provided an overall efficiency for charged track reconstruction of better than $95 \%$ and a momentum resolution which can be approximated by the following formula $\frac{\sigma_{p}}{p} \approx \frac{0.05}{\sqrt{L}} \oplus \frac{0.008 p}{\sqrt{L^{5}}}$, where the momentum $p$ is in $\mathrm{GeV} / c$ and the track length $L$ in $\mathrm{m}$. Reconstructed tracks were used to determine the event topology (the assignment of tracks to vertices), to reconstruct the vertex position and the track parameters at each vertex and, finally, to identify the vertex type (primary, secondary, etc.). A transition radiation detector (TRD) $[38,39]$ placed at the end of the active target was used for particle identification. Two scintillation counter trigger planes [40] were used to trigger on neutrino interactions in the NOMAD active target. A lead-glass electromagnetic calorimeter [41,
42] located downstream of the tracking region provided an energy resolution of $3.2 \% / \sqrt{E[\mathrm{GeV}]} \oplus 1 \%$ for electromagnetic showers and was crucial to measure the total energy flow in neutrino interactions. In addition, an iron absorber and a set of muon chambers located after the electromagnetic calorimeter was used for muon identification, providing a muon detection efficiency of $97 \%$ for momenta greater than $5 \mathrm{GeV} / c$.

The NOMAD neutrino beam consisted mainly of $v_{\mu}$ 's with an about $7 \%$ admixture of $\bar{v}_{\mu}$ and less than $1 \%$ of $v_{e}$ and $\bar{v}_{e}$. More details on the beam composition can be found in [30].

The main goal of the NOMAD experiment was the search for neutrino oscillations in a wide-band neutrino beam from 
the CERN SPS $[43,44]$. A very good quality of event reconstruction similar to that of bubble chamber experiments and a large data sample collected during four years of data taking (1995-1998) allow for detailed studies of neutrino interactions.

\subsection{Reconstruction of QEL events in the NOMAD detector}

A detailed information about the construction and performance of the NOMAD drift chambers as well as about the developed reconstruction algorithms is presented in [37]. Let us briefly describe some features relevant to the current QEL analysis. The muon track is in general easily reconstructed. However, when we study protons emitted in the $v_{\mu}$ QEL two-track candidates we deal with protons with momentum well below $1 \mathrm{GeV} / c$ and with emission angle above 60 degrees. For positive particles in the upward hemisphere of the NOMAD detector such conditions mean that these particles are almost immediately making a U-turn due to the magnetic field. There were no special efforts invested into tuning the NOMAD reconstruction program to reconstruct this particular configuration (which is rather difficult due to the fact that these protons are in the $1 / \beta^{2}$ region of ionization losses, traversing much larger amount of material, crossing drift cells at very large angles where the spacial resolution of the drift chambers is considerably worse and where a large amount of multiple hits is produced, etc.). Some of these effects are difficult to parameterize and to simulate at the level of the detector response in the MC simulation program. Thus, the reconstruction efficiencies for this particular configuration of outgoing protons could be different for the simulated events and real data.

Let us stress, however, that for protons emitted downwards we observed a good agreement between data and MC (see Fig. 6 below).

In the current analysis it was important to disentangle the reconstruction efficiency effects discussed above from the effects induced by intranuclear cascade (which could change the proton kinematics and thus introduce drastic changes in the final results due to the efficiency mismatch between simulated and real data). In order to get rid of an interplay between these two effects it was crucial to choose the region in the detector with a stable reconstruction efficiency. This could be achieved by selecting $v_{\mu}$ QEL events where protons are emitted in the lower hemisphere of the NOMAD detector. Therefore during the event selection we apply a cut on the muon to be emitted in the upper hemisphere (see Sect. 5).

The most upstream drift chamber was used as an additional veto to remove through-going muons from neutrino interactions upstream of the NOMAD active target. This is crucial for the study of single track events.

\section{Monte Carlo simulation of neutrino interactions and analysis procedure}

Inclusive (anti)neutrino charged-current (CC) and neutral current (NC) scattering can be considered as a mixture of several processes described by significantly different models. In our case, these are quasi-elastic scattering (QEL), single-pion production via intermediate resonance state (RES) and deep inelastic scattering (DIS). There is also a contribution from a coherent interaction of neutrino with a nucleus as a whole $(\mathrm{COH})$. Below we will describe in details the simulation scheme used for each of these processes and discuss the influence of the nuclear effects.

The overall procedure used to compute the QEL cross section and extract $M_{A}$ is as follows. Analytical expressions described in Sect. 4.6 are used to compute the numbers of produced background events. A Monte Carlo program, described below, is used to calculate the efficiencies for the various background sources to survive the selection procedure described in Sect. 5. These efficiencies are then applied to the numbers of produced events to calculate the background in our final sample and subtract it, yielding the numbers of QEL events. The QEL cross sections are extracted from them after correcting for the QEL efficiency. As a last step, the analytical expression for the QEL cross section is used to find the value of $M_{A}$ that best fits our results.

\subsection{Quasi-elastic neutrino scattering}

The standard representation of the weak hadronic current involved in the matrix elements of the processes $v_{\mu} n \rightarrow \mu^{-} p$ and $\bar{v}_{\mu} p \rightarrow \mu^{+} n$, is expressed in terms of 6 form factors, which in general are assumed to be complex [45]. They formally describe the hadronic structure and cannot be calculated analytically within the framework of the electro-weak interaction theory.

We neglect the second-class current contributions associated with the scalar and pseudo-tensor form factors. This is equivalent to the requirement of time reversal invariance of the matrix element (hence all form factors should be real functions of $Q^{2}$ ) and charge symmetry of the hadronic current (rotation about the second axis in the isotopic space).

The vector form factors $F_{V}$ and $F_{M}$ are related through the isospin symmetry hypothesis to the electromagnetic ones, which we will consider to be well known. Instead of the simple dipole parameterization, extensively used in previous experiments, we have chosen the Gari-Krüempelmann (GK) model [46] extended and fine-tuned by Lomon [47]. Specifically we explore the "GKex(05)" set of parameters [48] which fits the modern and consistent older data well and meets the requirements of dispersion relations and of QCD at low and high 4-momentum transfer [46]. 
For the axial and pseudo-scalar form factors we use the conventional representations [45]:

$F_{A}\left(Q^{2}\right)=F_{A}(0)\left(1+\frac{Q^{2}}{M_{A}^{2}}\right)^{-2}$

and

$F_{P}\left(Q^{2}\right)=\frac{2 m_{N}^{2}}{m_{\pi}^{2}+Q^{2}} F_{A}\left(Q^{2}\right)$,

where $F_{A}(0)=g_{A}=-1.2695 \pm 0.0029$ (measured in neutron $\beta$-decay [49]); $m_{\pi}$ and $m_{N}$ are the pion and nucleon masses.

As discussed in Sect. 2, the currently available experimental data on the axial mass $M_{A}$ allow for a wide spread. Thus, in our case, it should be considered as one of the available parameters, which can be used to adjust the MC simulation with the measured value of the total QEL cross section and observed distributions of the kinematic variables (other parameters, related to the modeling of the intranuclear cascade, will be described later).

Note that the expression for the pseudo-scalar form factor $F_{P}$ is nothing better than a plausible parameterization inspired by the PCAC hypothesis and the assumption that the pion pole dominates at $Q^{2} \lesssim m_{\pi}^{2}$ [45]. However, its contribution enters into the cross sections multiplied by a factor $\left(m_{\mu} / m_{N}\right)^{2}$. Hence, the importance of the related uncertainty is much reduced.

\subsection{Single-pion production through intermediate baryon resonances}

In order to describe the single-pion neutrino production through baryon resonances we adopt an extended version of the Rein and Sehgal model (RS) [50, 51], which seems to be one of the most widely trusted phenomenological approaches for calculating the RES cross sections. The generalization proposed in $[52,53]$ takes into account the final lepton mass and is based upon a covariant form of the charged leptonic current with definite lepton helicity. In our MC simulation we use the same set of 18 interfering nucleon resonances with masses below $2 \mathrm{GeV}$ as in [50] but with all relevant input parameters updated according to the current data $[49,54]$. Significant factors (normalization coefficients etc.), estimated in [50] numerically are recalculated by using the new data and a more accurate integration algorithm.

The relativistic quark model of Feynman, Kislinger, and Ravndal [55], adopted in the RS approach, unambiguously determines the structure of the transition amplitudes involved into the calculation and the only unknown structures are the vector and axial-vector transition form factors $G^{V, A}\left(Q^{2}\right)$. In [50] they are assumed to have the form

$$
\frac{G^{V, A}\left(Q^{2}\right)}{G^{V, A}(0)}=\left(1+\frac{Q^{2}}{4 m_{N}^{2}}\right)^{1 / 2-n}\left(1+\frac{Q^{2}}{M_{V, A}^{2}}\right)^{-2}
$$

where the integer $n$ in the first ("ad hoc") factor in (3) is the number of oscillation quanta of the intermediate resonance.

The vector mass $M_{V}$ is taken to be $0.84 \mathrm{GeV}$, that is the same as in the usual dipole parameterization of the nucleon electromagnetic form factors. The axial mass (which was fixed at $0.95 \mathrm{GeV}$ in the original RS paper) is set to the standard world averaged value $M_{A}=1.03 \mathrm{GeV}$. It is in good agreement with the results obtained in the recent analysis of the data from the BNL 7-foot deuterium filled bubble chamber: $M_{A}=1.08 \pm 0.07 \mathrm{GeV}$ [56]. Let us also note that the available experimental data for the single-pion neutrino production (as in the case of QEL scattering) does not permit a very definite conclusion about the value of the total RES cross section (and the corresponding axial mass value). The present uncertainties will be taken into account in the calculation of the systematic error of the current analysis.

To compensate for the difference between the $S U_{6}$ predicted value $(-5 / 3)$ and the experimental value for the nucleon axial-vector coupling $g_{A}$, Rein and Sehgal introduced a renormalization factor $Z=0.75$. In order to adjust the renormalization to the current world averaged value $g_{A}=$ -1.2695 [49] we have adopted $Z=0.762$. The harmonicoscillator constant $\Omega$, which accounts for the mass differences between states with different number of excitation quanta is set to its original value $\Omega=1.05 \mathrm{GeV}^{2}$.

Another essential ingredient of the RS approach is the non-resonant background (NRB). Its contribution is important in describing the existing data on the reactions $v_{\mu} n \rightarrow$ $\mu^{-} n \pi^{+}, v_{\mu} n \rightarrow \mu^{-} p \pi^{0}, \bar{v}_{\mu} p \rightarrow \mu^{+} p \pi^{-}$and $\bar{v}_{\mu} p \rightarrow$ $\mu^{+} n \pi^{0}$. In our Monte Carlo, the NRB is taken to come from the DIS part of the simulation. Therefore it has not been used in the RES part of our event generator.

\subsection{Deep inelastic scattering}

The MC simulation of the deep inelastic neutrino-nucleon scattering is based on the LEPTO 6.5.1 package [57] with several modifications [58, 59]. For hadronization we use the LUND string fragmentation model, as incorporated into the JETSET 7.4 program [60-62]. Structure functions are calculated at LO using the GRV98 [63] parton distribution functions.

Upon implementing the Monte Carlo for $v_{\mu}\left(\bar{v}_{\mu}\right)$ CC scattering, kinematic boundaries between exclusive (RES) and inclusive (DIS) channels must be defined. To avoid double counting, the phase space of the RES and DIS contributions should be separated by the conditions $W<W_{\text {cut }}^{\mathrm{RES}}$ and $W>W_{\text {cut }}^{\mathrm{DIS}}$, where $W$ is the invariant mass of the final hadronic system.

The maximum possible value for $W_{\text {cut }}^{\mathrm{RES}}$ is the upper limit of the RS model $(2 \mathrm{GeV})$, while inelastic scattering can take place from the one-pion production threshold (note, however, that this value is too small in principle since the struc- 
ture functions used in the calculation of the DIS cross section cannot be extrapolated down to this value).

Unfortunately, there is no clear physical recipe to determine exact numerical values for those cutoff parameters. The authors of GENIE MC code [64] adopt the value $W_{\text {cut }}^{\mathrm{RES}} \simeq W_{\text {cut }}^{\mathrm{DIS}} \sim 1.7 \mathrm{GeV}$. A comprehensive analysis of available experimental data made in $[65,66]$ suggests to decrease this cut to $\sim 1.5 \mathrm{GeV}$.

In the present analysis we set $W_{\text {cut }}^{\mathrm{RES}}=2 \mathrm{GeV}$ and $W_{\text {cut }}^{\mathrm{DIS}}=1.4 \mathrm{GeV}$. This choice allows for the non-resonant contribution to single-pion production to be accounted for by the DIS part of the Monte Carlo, providing e.g. $N\left(\mu^{-} p \pi^{0}\right.$ in DIS $) / N_{\text {dis }} \cdot \sigma_{\text {dis }} \approx \sigma\left(\mu^{-} p \pi^{0}\right.$ in NRB from RES), see previous subsection. Moreover, it is not at variance with experimental data as far as the total (anti)neutrino cross section is concerned.

\subsection{Coherent pion production}

In the processes described above, neutrinos interact with individual target nucleons. However, pions can be produced in a coherent interaction of the neutrino with the whole nucleus, i.e. in the case of $\mathrm{CC} v_{\mu}$ scattering $v_{\mu} \mathcal{N} \rightarrow \mu^{-} \pi^{+} \mathcal{N}$, where $\mathcal{N}$ is the target nucleus.

The details of the MC simulation can be found in [67], which is devoted to the investigation of this process in the NOMAD experiment. The flux-averaged cross section has been calculated following [68, 69] and has been estimated at $0.733 \times 10^{-38} \mathrm{~cm}^{2}$ per nucleus. For a recent experimental result at low incoming neutrino energy see [70]. Taking into account that the average mass number of the NOMAD target is 12.9, and using the number of recorded DIS events (see Sect. 6.1.1) one finds that the expected number of coherent pion production events is $\sim 2700$. Nevertheless, the probability for events of this type to be identified as QEL is $\sim 2 \%$ because of the small pion emission angle, so that the expected contamination of the selected QEL sample is lower than $0.4 \%$.

\subsection{Nuclear effects}

For typical NOMAD neutrino energies, we can assume that the incident neutrino interacts with one nucleon only inside the target nucleus, while the remaining nucleons are spectators (Impulse Approximation). In this case, one can describe the neutrino nucleus scattering by folding the usual expressions for the free neutrino-nucleon cross sections with a Fermi gas distribution.

In the relativistic Fermi gas model, the nucleus is considered as an infinite system of non-interacting nucleons. The phenomena related to the nuclear surface and to the interaction between nucleons can be taken into account by using a

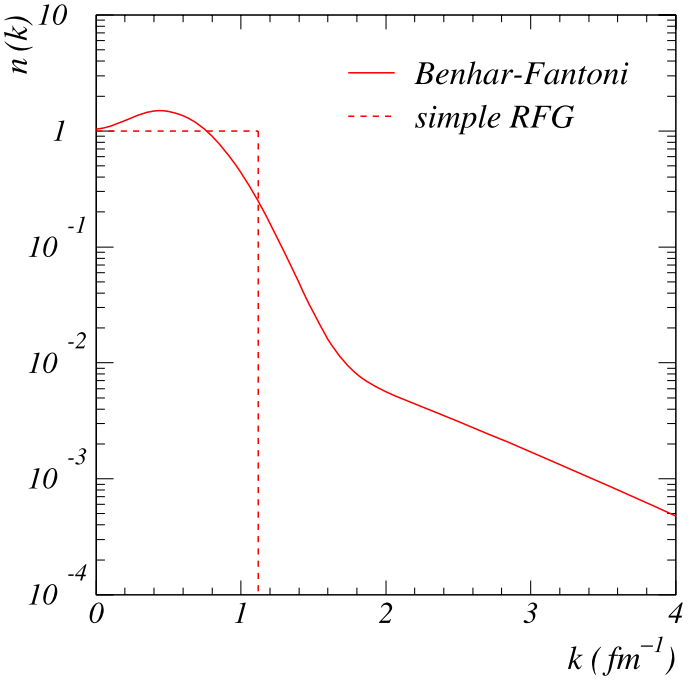

Fig. 2 Benhar-Fantoni parameterization [71] for the momentum distribution of the target nucleons (solid line), normalized to the Fermi distribution with zero temperature and Fermi momentum $P_{F}=221 \mathrm{MeV} / c$ (simple RFG, dashed line)

more realistic effective momentum distribution for the target nucleons. In the NOMAD event generator we used the Benhar-Fantoni parameterization [71], see Fig. 2.

The QEL simulation is based on the Smith-Moniz approach [72]. The momentum of the recoil nucleus and the nucleon binding energy are included in the conservation laws which determine the event kinematics. The only final state interaction (FSI) effect which is taken into account at this stage is the Pauli exclusion principle. The explicit form of the QEL differential cross section used in the MC code can be found in [32].

MC implementation of the Fermi gas model in the case of single-pion production is more straightforward. First, we generate the momentum of the target nucleon and make a Lorentz boost to its rest frame where the RES event can be simulated according to the extended RS model described in Sect. 4.2. The effect of Pauli blocking on the outgoing nucleon is taken into account as it is in the QEL MC.

In the case of the DIS neutrino scattering there are several specific nuclear effects (such as nuclear shadowing, pion excess and off-shell corrections to bound nucleon structure functions). They are described in the theoretical framework proposed in [73].

Simulating the re-interactions between particles produced at the primary neutrino collision off the target nucleon with the residual nucleus is an important ingredient of the $\mathrm{MC}$ event generator. To include this effect, commonly called final state interactions, we use the DPMJET package [74].

The intranuclear re-interaction of the particles generated by the QEL, RES or DIS event generators can be described and simulated by the Formation Zone Intranuclear Cascade model $[75,76]$ implemented in DPMJET. Secondaries from 
the first collision are followed along straight trajectories and may induce in turn intranuclear cascade processes if they reach the end of their "formation zone" inside the target; otherwise they leave the nucleus without interacting.

There are two important parameters in DPMJET. The first one, called the formation time $\tau_{0}$, controls the development of the intranuclear cascade. With increasing $\tau_{0}$, the number of cascade generations and the number of low-energy particles will be reduced. Its default value is $\tau_{0}=2.0$. After some tuning described below we adopted the value $\tau_{0}=1.0$ in our simulation of QEL and RES events.

Inside DPMJET, the momenta of the spectator nucleons are sampled from the zero temperature Fermi distribution. However, the nuclear surface effects and the interaction between nucleons result in a reduction of the Fermi momentum, see Fig. 2. It can be accounted for by introducing a correction factor $\alpha_{\text {mod }}^{F}$ (default value 0.6). Moreover, $\alpha_{\text {mod }}^{F}$ provides the possibility of some modification of the momentum distribution for the emitted low-energy nucleons.

At the end of the intranuclear cascade, the residual nucleus is supposed to go through some de-excitation mechanisms. It can be disrupted into two or more fragments, emit photons, nucleons or light particles (like $d, \alpha,{ }^{3} \mathrm{H},{ }^{3} \mathrm{He}$ ). We can easily neglect this contribution, since the typical energy of those particles is below the observation threshold of the NOMAD detector.

In our analysis, special attention will be devoted to the dependence of the obtained results on the intranuclear cascade parameters. As a cross-check, we compare our MC simulation for the QEL process with the predictions of the NUANCE event generator [77], which is currently used in a large number of neutrino experiments and which contains a different approach to the modeling of FSI effects.

\subsection{Analytical models used to fit the data}

In this subsection we estimate the number of signal quasielastic events in the initial $v_{\mu}\left(\bar{v}_{\mu}\right)$ CC sample.

The contribution of each process to the total set of events is proportional to its flux-averaged cross section:

$$
\langle\sigma\rangle=\int \sigma\left(E_{v}\right) \Phi\left(E_{\nu}\right) d E_{v} / \int \Phi\left(E_{v}\right) d E_{\nu}
$$

where

$\sigma\left(E_{v}\right)=n_{n} \sigma_{v n}\left(E_{v}\right)+n_{p} \sigma_{v p}\left(E_{v}\right)$

is the theoretical prediction for the cross section of the process at stake, $\Phi\left(E_{v}\right)$ denotes the NOMAD (anti)neutrino energy spectrum ${ }^{2}$ (see Fig. 3 ); $n_{n}\left(n_{p}\right)$ is the relative fraction of neutrons (protons) in the NOMAD target (see Sect. 3).

\footnotetext{
${ }^{2}$ The procedure used for the calculation of the flux and composition of the CERN SPS neutrino beam is described in [30].
}

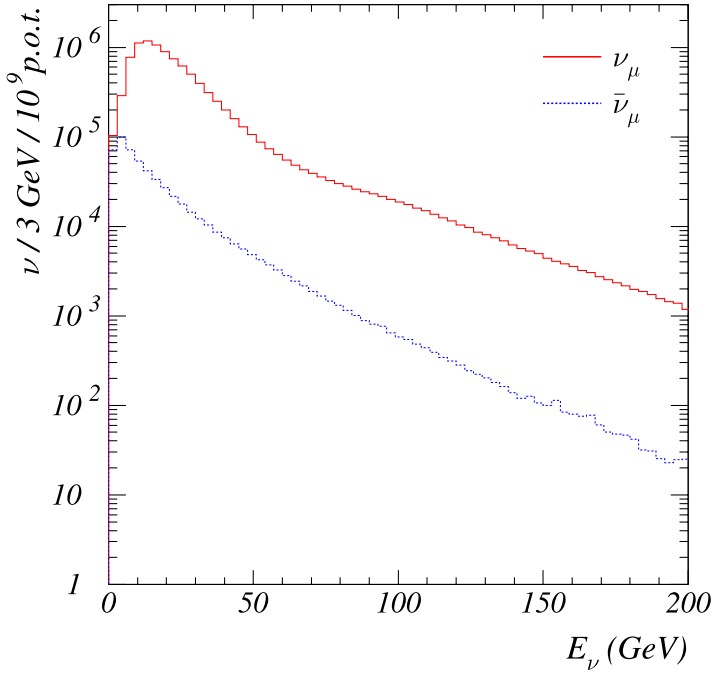

Fig. 3 The predicted energy spectra of $v_{\mu}$ and $\bar{v}_{\mu}$ at NOMAD for the transverse fiducial area of $100 \times 100 \mathrm{~cm}^{2}$

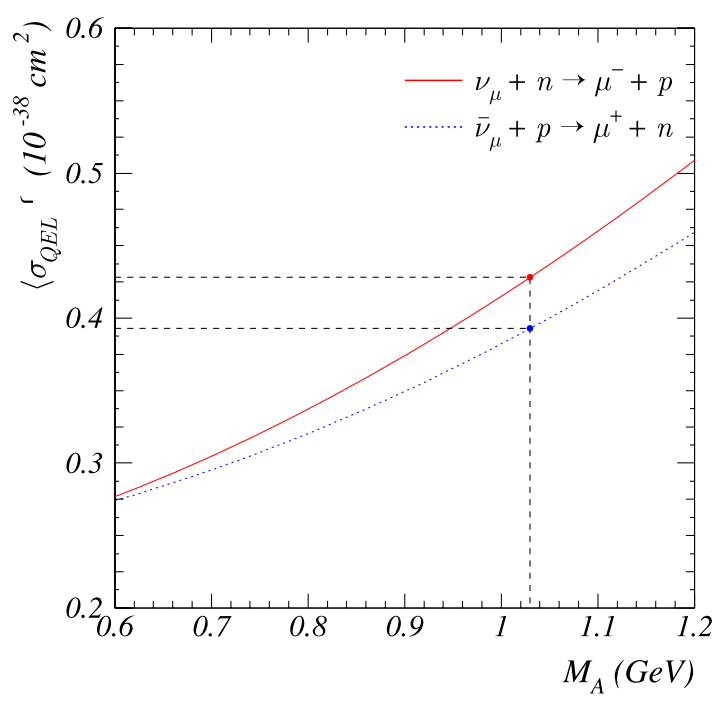

Fig. 4 Flux-averaged cross section of QEL (anti)neutrino scattering for the NOMAD $v_{\mu}\left(\bar{v}_{\mu}\right)$ beam as a function of the axial mass $M_{A}$

The QEL cross section was calculated in the framework of the Smith and Moniz model [72] for carbon with binding energy $E_{b}=25.6 \mathrm{MeV}$ and Fermi momentum $P_{F}=$ $221 \mathrm{MeV} / c$. No final state interactions are taken into account apart from Pauli blocking. As noted above, the final result depends strongly on the axial mass $M_{A}$ (see Fig. 4).

To estimate the RES contribution, we fold the extended RS model [53] for a free nucleon with the Pauli factor from [79]. The computation of $\sigma_{\mathrm{dis}}\left(E_{v}\right)$ has been done with the GRV98-LO PDF model as indicated in [65]. The cutoff parameters $W_{\text {cut }}^{\mathrm{RES}}$ and $W_{\text {cut }}^{\mathrm{DIS}}$ are the same as for the MC simulation. The analytical calculations do not include nu- 


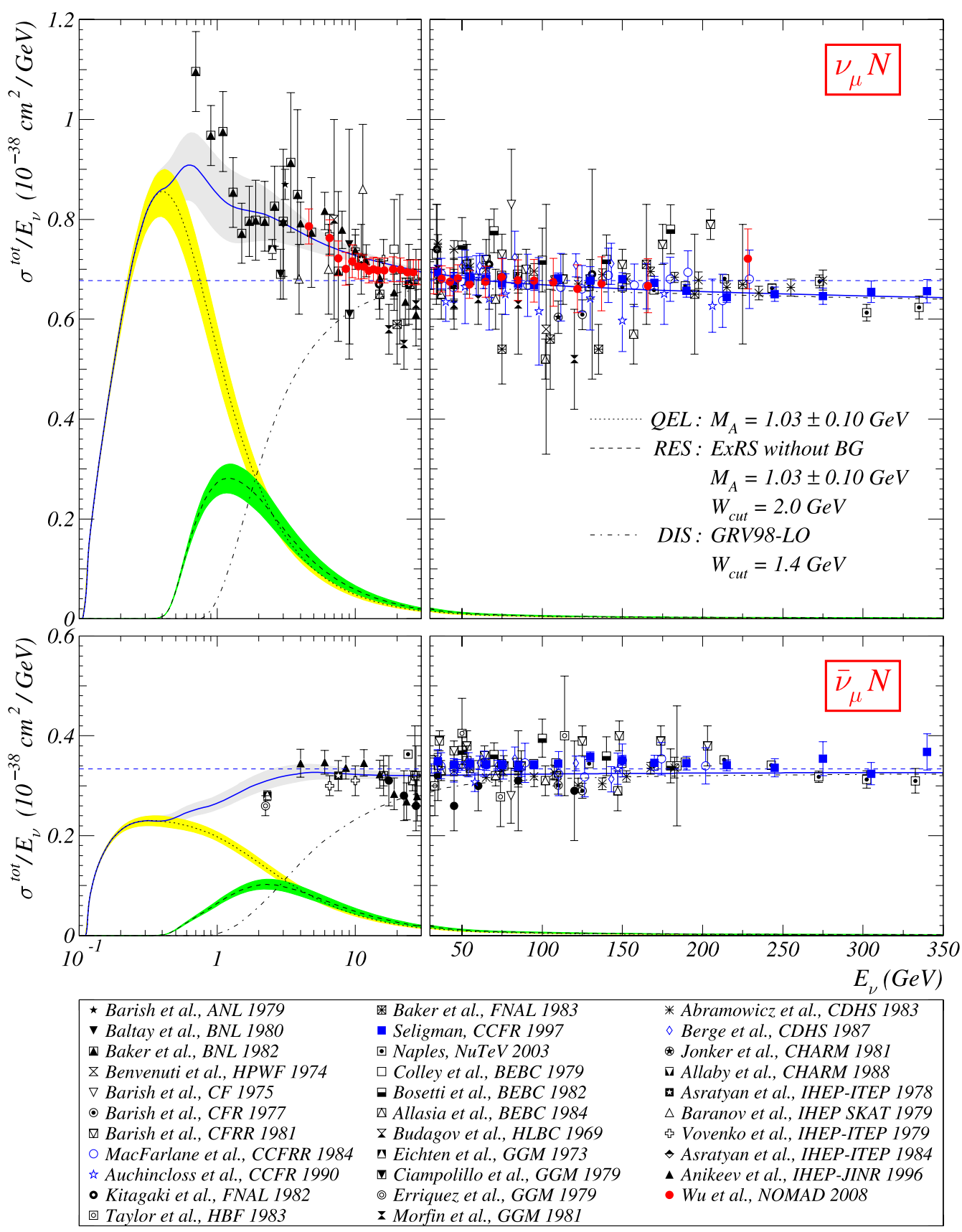

Fig. 5 Slopes of the total $v_{\mu}$ and $\bar{v}_{\mu}$ CC scattering cross sections off an isoscalar nucleon. Experimental data come from [65] and from [78] for the NOMAD points. The smooth curves show the QEL, RES, and DIS contributions and their sums calculated as described in Sect. 4.6. RES and DIS are used in this analysis to subtract background from experimental data (see (11)). The axial mass $M_{A}$, which enters both QEL and RES, is given the value used in

clear corrections and final state interactions apart from Pauli blocking.

The results from these calculations are shown as curves in Fig. 5 which also includes a compendium of total (anti)neutrino cross-section data. our Monte-Carlo. The bands reflect a $\pm 10 \%$ variation around it, roughly spanning the core of the hitherto published measurements. Our final result for $M_{A}$ is 2-3\% above the MC value (see (25)-(27)). Also shown are the energy averaged slopes published by the Particle Data Group [49]: $(0.677 \pm 0.014) \times 10^{-38} \mathrm{~cm}^{2} / \mathrm{GeV}$ for $v_{\mu} N$ and $(0.334 \pm 0.008) \times 10^{-38} \mathrm{~cm}^{2} / \mathrm{GeV}$ for $\bar{v}_{\mu} N$

Table 3 contains our results for the reduced fiducial volume of the NOMAD detector: $|X, Y| \leqslant 100 \mathrm{~cm}$; the average $v_{\mu}\left(\bar{v}_{\mu}\right)$ energy was 25.9 (17.6) GeV.

Combining all these, the expected fraction of quasielastic events in the initial $v_{\mu}\left(\bar{v}_{\mu}\right) \mathrm{CC}$ sample before any 
Table 3 Flux-averaged cross sections of the QEL, RES, DIS CC and NC processes per one nucleon of the NOMAD target. Neutrino beam spectrum corresponds to the $|X, Y| \leqslant 100 \mathrm{~cm}$ fiducial area. The unit used for the cross section is $10^{-38} \mathrm{~cm}^{2}$

\begin{tabular}{lrc}
\hline Process type & \multicolumn{1}{c}{$v_{\mu}$} & $\bar{v}_{\mu}$ \\
\hline QEL & 0.428 & 0.393 \\
RES & 0.576 & 0.432 \\
DIS CC & 16.643 & 4.876 \\
DIS NC & 5.335 & \\
\hline
\end{tabular}

special selection is about $2.4 \%(6.9 \%)$ or $\sim 20300(\sim 1360)$ events.

\section{Events selection}

In this section we describe particular features of reconstruction and identification of $v_{\mu}$ and $\bar{v}_{\mu}$ QEL events.

\section{$5.1 v_{\mu} n \rightarrow \mu^{-} p$ selection}

For a $v_{\mu} n \rightarrow \mu^{-} p$ event one can expect two tracks originating from the reconstructed primary vertex: ${ }^{3}$ one of them should be identified as a muon, while the second track is assumed to be a proton. Later we shall refer to events with such a topology as 2-track events. ${ }^{4}$

Sometimes the proton track cannot be reconstructed, e.g. if its momentum is below the detector registration threshold. In this case, we deal with only one muon track and we call such an event a 1-track event.

The expected ratio between 1-track and 2-track events for the pure standard QEL MC sample is $54.3 \%: 45.7 \%$.

There are three possible reasons for the reconstruction of the proton track in a QEL event to fail:

- the proton, which was born in the neutrino interaction with the target nucleon, has too low a momentum or too large an emission angle (this depends on the parameters of the model used to describe the neutrino-nucleon interaction, in particular, on the value of the axial mass);

- the proton from the primary neutrino interaction was involved in an intranuclear cascade and lost part of its energy (this is controlled by the DPMJET parameters, mainly by the formation time $\tau_{0}$ );

\footnotetext{
${ }^{3}$ All charged tracks originating within a $5 \mathrm{~cm}$ box around the reconstructed primary vertex are forced to be included into it; we have also tried to vary this parameter by enlarging the size of the box to $10 \mathrm{~cm}$ and found that the final results are rather stable (within $0.3 \%$ for the measured QEL cross section).

${ }^{4}$ In this analysis we do not take into account clusters in the electromagnetic calorimeter, which can be associated with neutral particles, originating from the primary vertex.
}

- the detector magnetic field deviates positively charged particles upwards; therefore, if a slow proton is emitted at an azimuth $\varphi_{h} \sim \pi / 2$, its trajectory is almost parallel to the drift chamber planes and its track reconstruction efficiency (which depends on the number of hits associated with the track) is significantly lower than in the case of a proton emitted downwards at $\varphi_{h} \sim 3 \pi / 2$.

In Fig. 6 (left) we illustrate these last two effects: the magnetic field is the cause of the asymmetry in the azimutal distribution of the reconstructed protons, while varying the formation time parameter $\tau_{0}$ affects the expected number of tracks uniformly.

In Fig. 7 we display an example of distributions of the leading proton momentum $p_{h}$ and emission angle $\theta_{h}$ before and after FSI for the QEL neutrino scattering. The proton reconstruction probabilities are also shown as functions of $p_{h}$ and $\theta_{h}$ : one can observe a fast decrease at low proton momenta (below $300 \mathrm{MeV} / c$ ) and large emission angles (larger than $72^{\circ}$ ). So, FSI tends to increase the fraction of events in kinematic domains with low proton reconstruction efficiency and therefore to change the expected fraction of events with a given topology in the identified QEL sample.

Using 2-track events only for the analysis may seem very attractive, since we could significantly reduce the background contamination with the help of additional kinematic variables (details can be found below). However, the results thus obtained might still have large systematic uncertainties coming from insufficient understanding of nuclear effects.

The QEL events which are not reconstructed as 2-track events will populate mainly the 1 -track sample. But $\sigma_{\mathrm{qel}}$ extracted from this sample will suffer from the same source of uncertainty. However, the measurement of the QEL cross section simultaneously from both samples is expected to have only little dependence on the uncertainties in the modeling of FSI effects and this is indeed what is found in the data (see Sect. 8).

Therefore, the strategy of our analysis (selection criteria) in the case of $v_{\mu} n \rightarrow \mu^{-} p$ can be outlined as follows.

- Fiducial volume cut. The reconstructed primary vertex should be within the restricted ${ }^{5}$ fiducial volume $(\mathrm{FV})$ :

$$
|X, Y| \leqslant 100 \mathrm{~cm}, \quad 25 \leqslant Z \leqslant 395 \mathrm{~cm}
$$

- Identified mиon. We require the presence of a reconstructed and identified negatively or positively charged muon for the neutrino and antineutrino analyses respectively. In order to avoid possible problems with detector reconstruction inefficiencies (see discussion in Sect. 3.1), we require $0<\varphi_{\mu}<\pi$, where $\varphi_{\mu}$ is the muon azimuthal

\footnotetext{
${ }^{5}$ We use a more stringent cut $Z>50 \mathrm{~cm}$ for the data collected during 97 and 98 , when the first drift chamber module was substituted by the NOMAD STAR detector.
} 


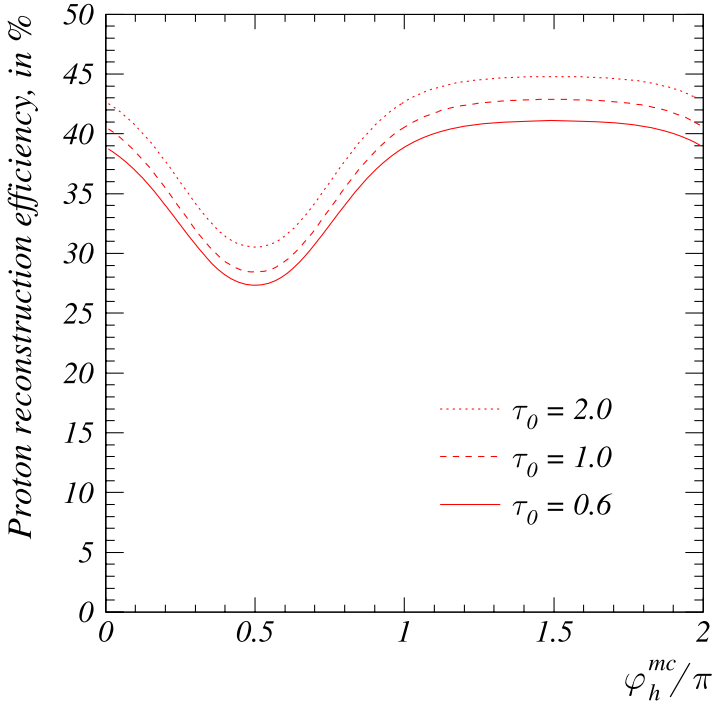

Fig. 6 Reconstruction efficiency of the proton track as a function of its azimuth $\varphi_{h}$ for $v_{\mu}$ QEL scattering; the curves are smoothed MC predictions obtained for different values of the formation time $\tau_{0}$ (left). Comparison of the muon azimuth $\varphi_{\mu}$ distributions in data and MC

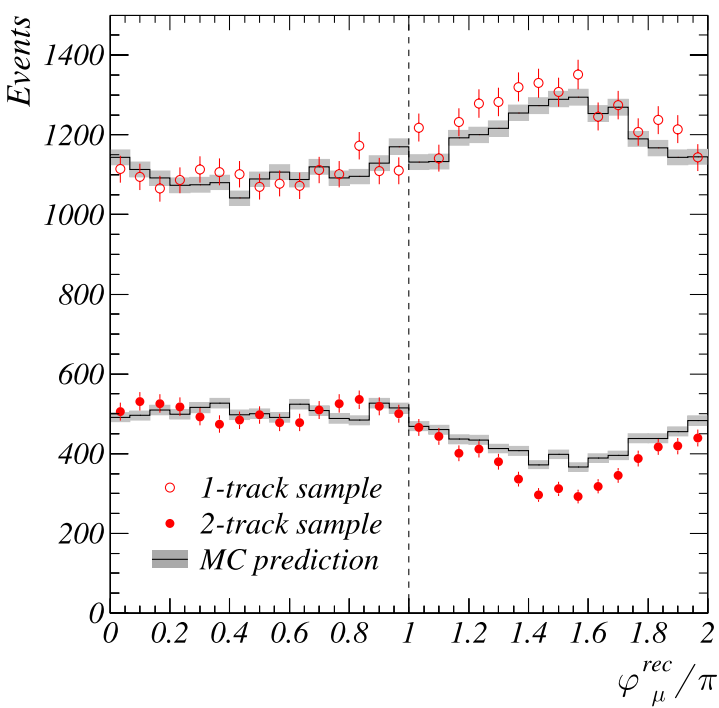

for 1-track and 2-track samples (right); the vertical dashed line corresponds to the cut $0<\varphi_{\mu}<\pi$ which was applied during the event selection

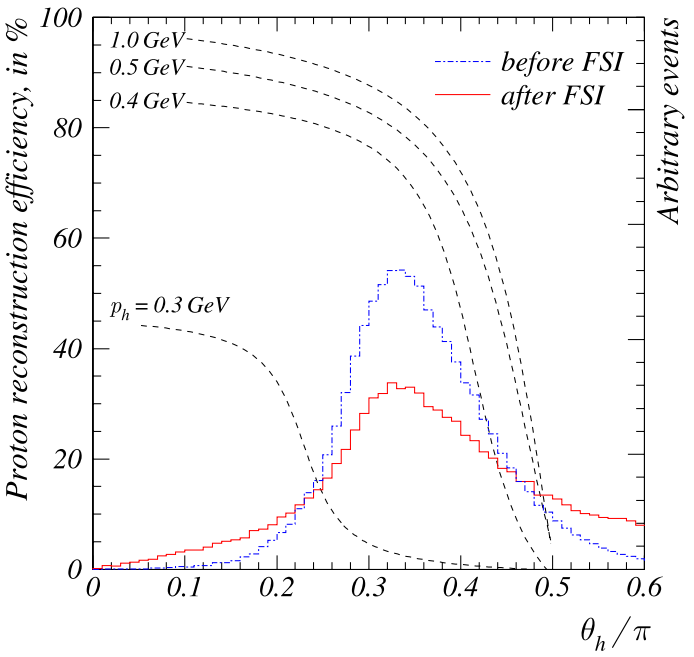

Fig. 7 Distribution of the leading proton momentum (left) and emission angle (right) before (dash-dotted line) and after (solid line) FSI simulation. Dashed lines show the proton reconstruction efficiency as a function of the proton momentum and emission angle (for $\pi<\varphi_{h}<2 \pi$ )

angle (so, the proton track should lie in the bottom hemisphere), see Fig. 6 (right).

This choice is validated by our final errors being dominated by systematics as will be shown below.

- Event topology and reconstructed kinematic variables. We assign the events to the 1-track and 2-track subsamples and calculate $E_{v}$ and $Q^{2}$.

- Single track sample (only one charged lepton is reconstructed and identified). To avoid contamination from the through-going muons we extrapolate the muon track to the first drift chamber and require the absence of veto chamber hits in the vicinity of the intersection point. The effectiveness of this quality cut was controlled by visual scanning of the reconstructed 1-track events in the experimental data. Another quality cut was used to suppress a possible contribution from inverse muon decay events: we require the muon transverse momentum to be greater than $0.2 \mathrm{GeV} / c$ (see Sect. 6.1 for more details).

The kinematic variables are reconstructed under the assumption that the target nucleon is at rest. For the 1-track events, the muon momentum and direction are the sole measurements and we have to use the conser- 
vation laws (assuming QEL) to compute other kinematic quantities:

$$
\begin{aligned}
& E_{v}=\frac{M E_{\mu}-m_{\mu}^{2} / 2}{M-E_{\mu}+p_{\mu} \cos \theta_{\mu}}, \\
& Q^{2}=2 M\left(E_{v}-E_{\mu}\right), \\
& p_{h}=\left(\left(E_{\nu}-p_{\mu} \cos \theta_{\mu}\right)^{2}+p_{\mu}^{2} \sin ^{2} \theta_{\mu}\right)^{1 / 2}, \\
& \cos \theta_{h}=\left(E_{\nu}-p_{\mu} \cos \theta_{\mu}\right) / p_{h},
\end{aligned}
$$

where $p_{\mu}, \theta_{\mu}\left(p_{h}, \theta_{h}\right)$ are the momentum and emission angle of the outgoing muon (nucleon); see Fig. 8. We note that for the neutrino energies relevant for this analysis (above $3 \mathrm{GeV}$ ) there is no difference between

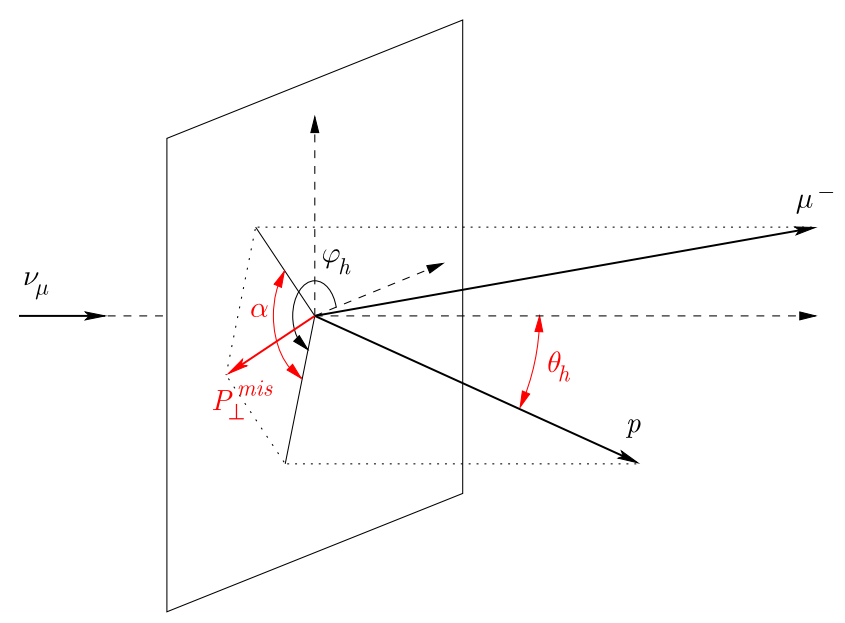

Fig. 8 Likelihood variables: missing transverse momentum $P_{\perp}^{\mathrm{mis}}$, proton emission angle $\theta_{h}$, angle $\alpha$ between the transverse components of the charged tracks

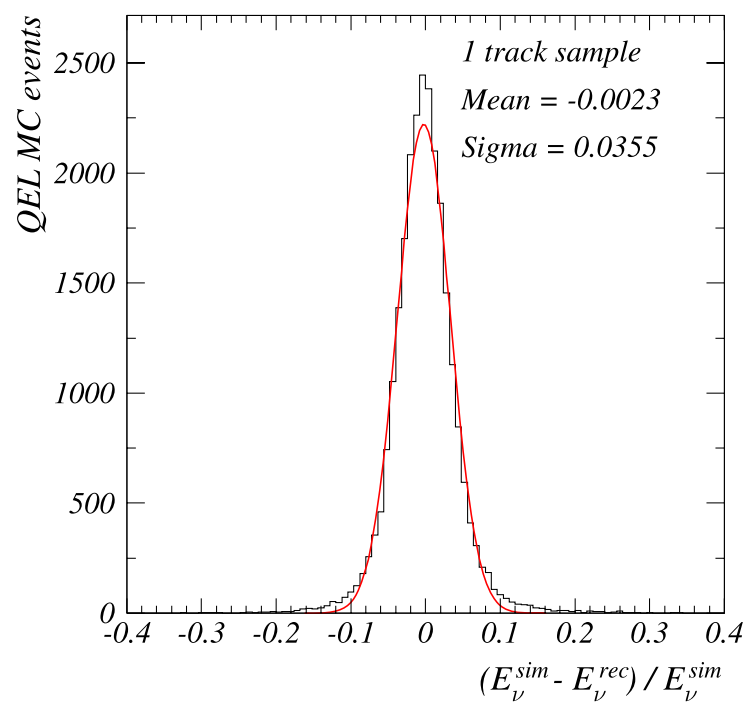

the calculations based on the approximated formulae above and the precise one, which takes into account the binding energy (see e.g. (4) in [26]). With the help of the MC simulation we estimate the resolution of the reconstructed $E_{v}$ and $Q^{2}$ as $3.6 \%$ and $7.8 \%$ respectively.

- Two track sample (both the negative muon and the positively charged track are reconstructed). For a reliable reconstruction, we require that the number of hits associated with the positively charged track should be greater than 7 and its momentum $p_{h}>300 \mathrm{MeV} / c$. Otherwise such an event is downgraded to the 1-track sample.

For 2-track events, we use both the muon and the proton reconstructed momenta to estimate $E_{v}$ and $Q^{2}$ :

$$
\begin{aligned}
& E_{v}=p_{\mu} \cos \theta_{\mu}+p_{h} \cos \theta_{h}, \\
& Q^{2}=2 E_{v}\left(E_{\mu}-p_{\mu} \cos \theta_{\mu}\right)-m_{\mu}^{2} .
\end{aligned}
$$

The expected resolutions for $E_{v}$ and $Q^{2}$ are $3.6 \%$ and $7.1 \%$.

The quality of the neutrino energy $E_{v}$ reconstruction for 1- and 2-track samples is illustrated in Fig. 9. It was checked that for the 2-track sample the derived cross sections are consistent within errors for both methods of $E_{v}$ calculation.

- Background suppression. The contamination from RES and DIS processes can be suppressed by using the difference between kinematical distributions in the QEL and background events as well as by the identification of the reconstructed positively charged track as a proton (for the 2-track sample only). Therefore we apply the following.

- Identification of the positively charged track.

The momentum-range method [80] can be reliably applied for low energy protons since their tracks are

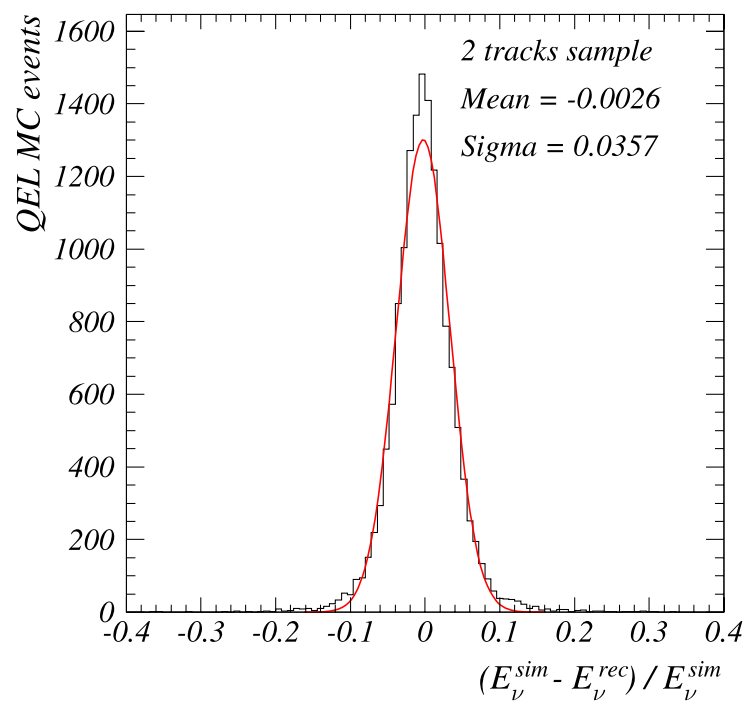

Fig. 9 The quality of the neutrino energy $E_{v}$ reconstruction for 1- and 2-track samples 

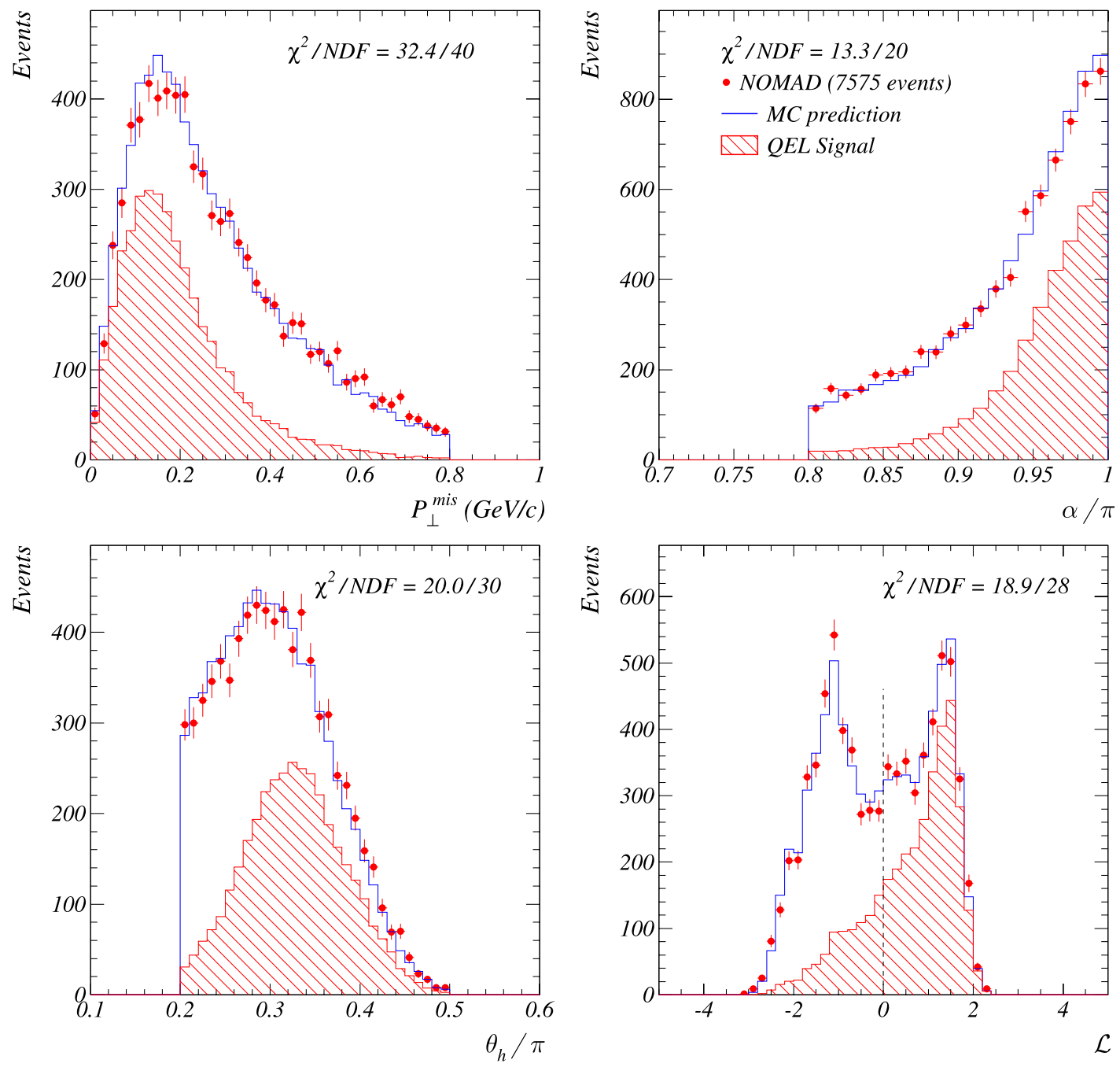

Fig. 10 The $P_{\perp}^{\text {mis }}, \alpha, \theta_{h}$ and likelihood distributions for a mixture of QEL, RES and DIS simulated events (histograms) compared to real data (points with error bars). The MC distributions are normalized to the number of events observed in the data

shorter compared to that of $\pi^{+}$(the main background for proton identification) due to larger ionization losses. In our case, this method can be applied to about $17 \%$ of the events. ${ }^{6}$

\section{- Kinematical criteria.}

In the case of the 2-track sample, we can use additional kinematic variables to suppress background con-

\footnotetext{
${ }^{6} \mathrm{We}$ also undertook an attempt to identify positively charged particles using the TRD information. A special algorithm [81, 82] can be potentially used for discrimination between two particle-ID hypotheses $(p / \pi$ in our case). However, a low momentum $(\sim 0.9 \mathrm{GeV})$ of the particle and a rather large emission angle $\left(\gtrsim 45^{\circ}\right)$ result in that either the particle does not reach the TRD or the number of residual TRD hits is not large enough for the identification. Therefore, the TRD algorithm could be applied only to a limited fraction of events $(\sim 6 \%)$ and cannot play any significant role in our analysis.
}

tamination. We build the likelihood ratio

$\mathcal{L}=\ln \frac{P(\ell \mid \mathrm{QEL})}{P(\ell \mid \mathrm{BG})}$,

using 3-dimensional correlations between the following kinematic variables (see Fig. 8): the missing transverse momentum $P_{\perp}^{\text {mis }}$, the proton emission angle $\theta_{h}$, and the angle $\alpha$ between the transverse components of the charged primary tracks. The following precuts were applied prior to the likelihood construction: $P_{\perp}^{\mathrm{mis}}<0.8 \mathrm{GeV} / c, 0.2 \leqslant \theta_{h} / \pi \leqslant 0.5$ and $\alpha / \pi \geqslant 0.8$.

In (7) the $P(\ell \mid \mathrm{QEL})$ and $P(\ell \mid \mathrm{BG})$ are the probabilities for signal and background events to have the values of the variables $\ell=\left(P_{\perp}^{\text {mis }}, \theta_{h}, \alpha\right)$. We have found that the DIS and RES probability functions are very similar; therefore we build the likelihood function taking only resonance events for the denominator of (7). 
Table 4 Number of data $N_{\text {data }}$ and renormalized MC $N_{\text {mc }}$ events in $v_{\mu}$ and $\bar{v}_{\mu}$ QEL samples; expected selection efficiency, purity and background contaminations (BG) for different stages of the analysis. Num- ber of MC events $N_{\mathrm{mc}}$ were normalized to the $N_{\mathrm{data}}$ after the selection. Expected numbers of MC events were recalculated for the earlier stages of the analysis on the basis of this number

\begin{tabular}{|c|c|c|c|c|c|c|c|}
\hline & QEL eff. (\%) & QEL purity (\%) & RES BG (\%) & DIS BG (\%) & oth. BG (\%) & $N_{\text {data }}$ & $N_{\mathrm{mc}}$ \\
\hline$v_{\mu}$ 1-track before $\theta_{h}$ cut & 23.7 & 29.0 & 18.3 & 52.3 & 0.4 & 16508 & 16633.7 \\
\hline$v_{\mu} 1$-track after $\theta_{h}$ cut & 21.3 & 41.7 & 23.2 & 34.5 & 0.6 & 10358 & 10358.0 \\
\hline$v_{\mu} 2$-track after quality cuts & 21.2 & 9.4 & 9.3 & 77.9 & 3.4 & 41768 & 44937.2 \\
\hline$v_{\mu} 2$-track after $\theta_{h}$ cut & 18.4 & 36.2 & 18.0 & 45.6 & 0.2 & 10144 & 10355.6 \\
\hline$v_{\mu}$ 2-track after $P_{\perp}^{\mathrm{mis}}$ cut & 18.2 & 40.8 & 18.8 & 40.1 & 0.3 & 9084 & 9082.8 \\
\hline$v_{\mu}$ 2-track after $\alpha$ cut & 17.6 & 47.2 & 17.3 & 35.2 & 0.3 & 7575 & 7609.0 \\
\hline$v_{\mu} 2$-track after $\mathcal{L}$ cut & 13.3 & 73.9 & 10.2 & 15.8 & $<0.1$ & 3663 & 3663.0 \\
\hline $\bar{v}_{\mu}$ 1-track before $\theta_{h}$ cut & 81.8 & 29.8 & 22.8 & 45.8 & 1.6 & 3585 & 3555.8 \\
\hline $\bar{v}_{\mu} 1$-track after $\theta_{h}$ cut & 64.4 & 36.6 & 28.5 & 33.6 & 1.3 & 2237 & 2237.0 \\
\hline
\end{tabular}
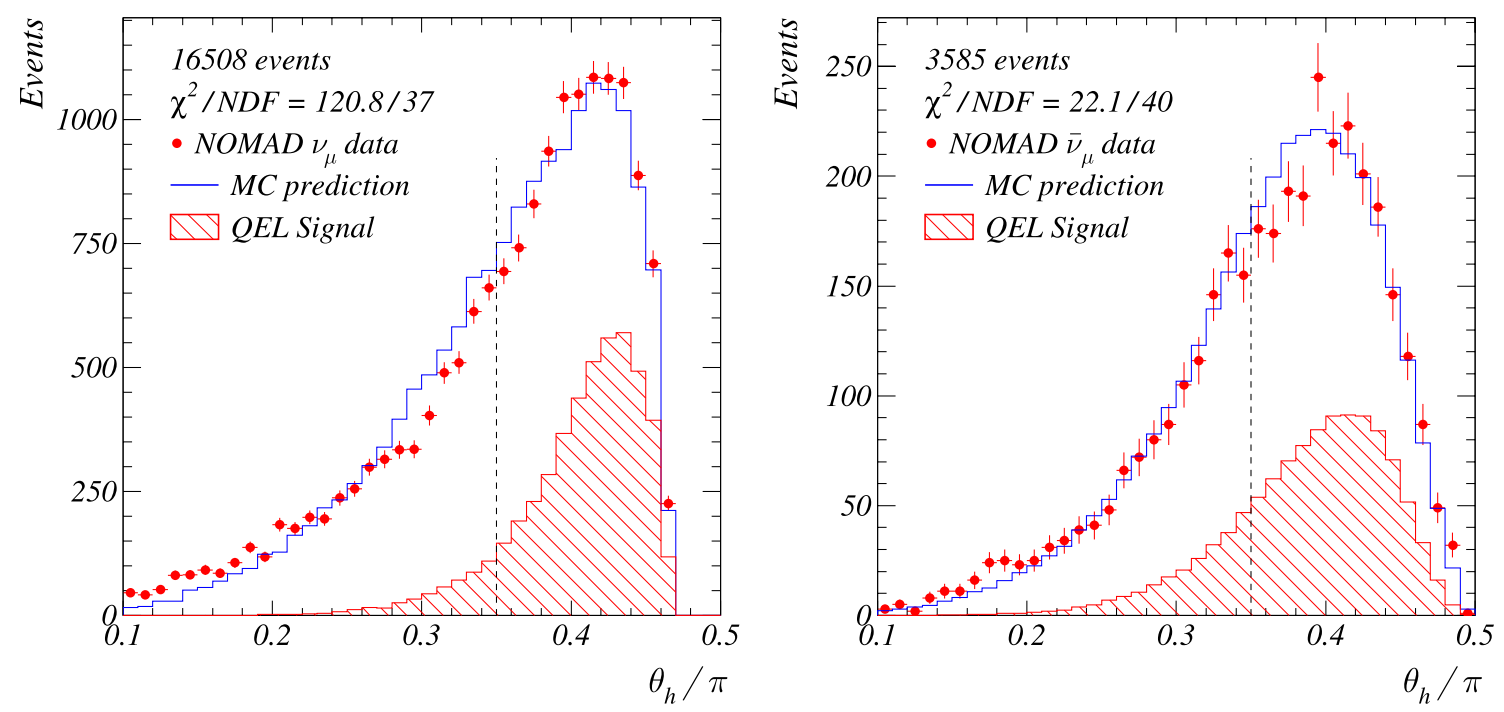

Fig. 11 The $\theta_{h}$ distributions for single track $v_{\mu}$ (left) and $\bar{v}_{\mu}$ (right) samples: comparison of MC distributions (histograms) with the real data (points with error bars)

The comparison of $P_{\perp}^{\text {mis }}, \alpha, \theta_{h}$ and $\mathcal{L}$ distributions in the data with the mixture of simulated QEL, RES and DIS events weighted by their flux-averaged cross sections is displayed in Fig. 10. The good agreement observed between MC predictions and experimental data confirms a reasonable understanding of the background contaminations and reconstruction efficiency in our analysis. For example, after the likelihood cut, the respective contributions of QEL, RES, DIS and $\mathrm{COH}$ given by the cross sections and the efficiencies computed with the help of the MC for each process separately are as specified on the corresponding $\mathcal{L}>0$ line in Table 4. With the reduction factors for the $\mathcal{L}>0$ cut as given by the MC in the various channels, and normalizing the total $\mathrm{MC}$ to the data at this last stage, we find a total of 7609 events before the cut, distrib- uted as shown by the figures on the corresponding line. The excess of $34 \mathrm{MC}$ events relative to the data, which are necessarily mostly background, can be taken as evidence that there is less than $1 \%$ excess background in the MC after the pre-cuts. Since the total MC background is of the order of 4000 events, the number found is well under the expected statistical fluctuations. Therefore, there is no evidence of a statistically significant discrepancy.

In the case of 1-track events, our abilities to suppress background contamination are limited since all kinematic variables are expressed in terms of the muon momentum $p_{\mu}$ and emission angle $\theta_{\mu}$ with the help of the conservation laws for QEL events. Therefore, the proton reconstructed emission angle, (6), can be con- 


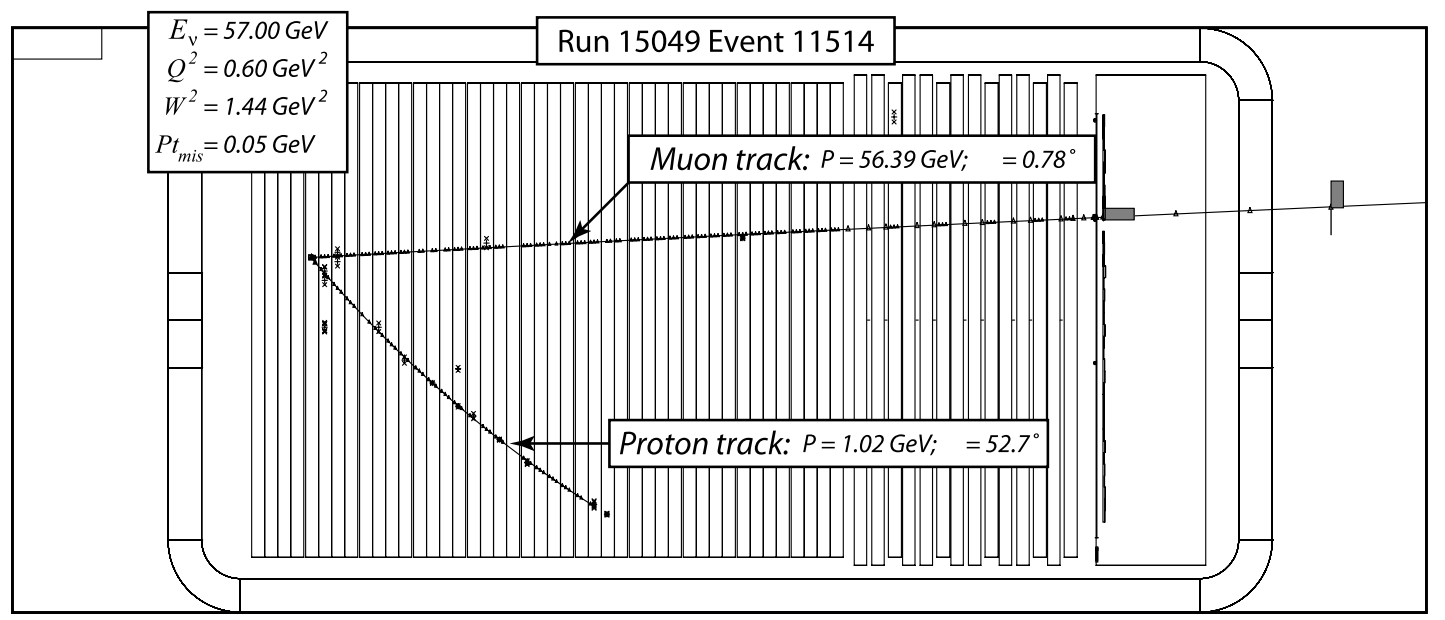

Fig. 12 A typical example of data event (run 15049 event 11514) identified as $v_{\mu} n \rightarrow \mu^{-} p$ in this analysis. The long track is identified as a muon, the short track is assumed to be a proton

sidered as an analog of the likelihood function (see Fig. 11).

The explicit values for the kinematic selection criteria $\left(\mathcal{L} \geqslant 0\right.$ for the 2 -track sample and $0.35 \leqslant \theta_{h} / \pi \leqslant$ 0.5 for the 1-track sample) were found from the optimization of the sensitivity $\mathrm{SG} / \sqrt{\mathrm{SG}+\mathrm{BG}}$, where SG and $\mathrm{BG}$ are the expected numbers of signal and background events in the identified QEL sample.

\section{$5.2 \bar{v}_{\mu} p \rightarrow \mu^{+} n$ selection}

The investigation of the antineutrino sample is a much simpler task since these events are mostly ( $\sim 96 \%$ of cases) reconstructed as 1-track events (we have no hits from outgoing neutrons in the drift chambers). Therefore, we require identification of the positively charged muon and follow the procedure for the 1-track sample discussed above. The only difference is the absence of contamination from the inverse muon decay events, so we do not need to apply the quality cut on the transverse muon momentum.

In Table 4 we summarize the information about the selection of samples with $v_{\mu} n \rightarrow \mu^{-} p$ and $\bar{v}_{\mu} p \rightarrow \mu^{+} n$ candidates in the data. The last two columns of this table allow us to make checks of compatibility between the levels of background in the data and in our simulations in a manner similar to what is explained above for the two track sample.

An example of the 2-track event from real data identified as $v_{\mu} n \rightarrow \mu^{-} p$ is displayed in Fig. 12.

\section{The QEL cross section and axial mass measurements}

In this section we describe our analysis procedure. The QEL cross section measurement using normalization either to the total (DIS) $v_{\mu}\left(\bar{v}_{\mu}\right)$ CC cross section or to the inverse muon decay (IMD), $v_{\mu} e^{-} \rightarrow \mu^{-} v_{e}$, events is first presented in Sect. 6.1. Afterwards, we describe the procedure used to extract the value of the axial mass $M_{A}$ from the fit of the $Q^{2}$ distribution. This is the subject of Sect. 6.2.

\subsection{The QEL cross-section measurement}

Since there was no precise knowledge of the integrated neutrino flux in the NOMAD experiment, we use a different process with a better known cross section, recorded at the same time, for the normalization of the QEL cross section. A similar procedure was often applied in previous neutrino experiments, as for example, CERN BEBC [18]. Moreover, the use of another process recorded in the same experimental runs allows one to reduce significantly the systematic uncertainty related to the detector material composition. Nevertheless, this auxiliary process must meet two requirements: its cross section should be measured with rather high accuracy and the corresponding events can easily be extracted from the full data sample.

Let us divide the investigated interval of neutrino energy into several bins and enumerate them with index $i=1 . . N_{E}$. Then, the number of identified QEL events in the $i$ th bin with boundaries $\left[E_{i}, E_{i+1}\right]$ is

$N_{i}^{\mathrm{dat}}=N_{i}^{\mathrm{bg}}+C \sum_{j=1}^{N_{E}} \varepsilon_{i j}^{\mathrm{qel}} \Phi_{j}\left\langle\sigma_{\mathrm{qel}}\right\rangle_{j}$,

where

$\Phi_{i}=\int_{E_{i}}^{E_{i+1}} \Phi(E) d E, \quad \sum_{i=1}^{N_{E}} \Phi_{i}=1$ 
and

$\left\langle\sigma_{\mathrm{qel}}\right\rangle_{i}=\frac{1}{\Phi_{i}} \int_{E_{i}}^{E_{i+1}} \sigma_{\mathrm{qel}}(E) \Phi(E) d E$.

The coefficient $C$ accumulates the absolute neutrino flux and the number of target nucleons. The matrix element $\varepsilon_{i j}^{\mathrm{qel}}$ is the probability that the reconstructed neutrino energy $E_{v}$ of a QEL event falls into the $i$ th bin, while the simulated energy actually belongs to the $j$ th bin.

The expected background contamination is

$N_{i}^{\mathrm{bg}}=C\left(\varepsilon_{i}^{\mathrm{res}}\left\langle\sigma_{\mathrm{res}}\right\rangle+\varepsilon_{i}^{\mathrm{dis}}\left\langle\sigma_{\mathrm{dis}}\right\rangle\right)$

where we use the definition of (4) for $\left\langle\sigma_{\mathrm{bg}}\right\rangle ; \varepsilon_{i}^{\mathrm{bg}}$ denotes the renormalized energy distribution in $\mathrm{BG}$ events passing the QEL identification procedure:

$\sum_{i=1}^{N_{E}} \varepsilon_{i}^{\mathrm{bg}}=\varepsilon^{\mathrm{bg}}=N_{\mathrm{rec}}^{\mathrm{bg}} / N_{\text {sim }}^{\mathrm{bg}}$.

Here $N_{\text {sim }}^{\text {bg }}$ and $N_{\text {rec }}^{\text {bg }}$ are the number of MC events simulated and identified as QEL in the chosen detector FV.

Similar equations can be written for any other process recorded in the same detector FV. If we identify $N_{0}$ events of a process, whose flux-averaged cross section in an energy interval containing these events is $\sigma_{0}$, we can write

$N_{0}=C \Phi_{0} \sigma_{0}$,

where $\Phi_{0}$ is the relative part of the neutrino flux belonging to the same energy interval. (we assume that $N_{0}$ is background subtracted and efficiency corrected).

We can now get rid of $C$ and write the final equation for $\left\langle\sigma_{\mathrm{qel}}\right\rangle_{i}$ :

$$
\begin{aligned}
\left\langle\sigma_{\mathrm{qel}}\right\rangle_{i}= & \frac{1}{\Phi_{i}} \sum_{j=1}^{N_{E}}\left(\varepsilon_{\mathrm{qel}}^{-1}\right)_{i j} \\
& \times\left[N_{j}^{\mathrm{dat}} \frac{\Phi_{0} \sigma_{0}}{N_{0}}-\varepsilon_{j}^{\mathrm{res}}\left\langle\sigma_{\mathrm{res}}\right\rangle-\varepsilon_{j}^{\mathrm{dis}}\left\langle\sigma_{\mathrm{dis}}\right\rangle\right] .
\end{aligned}
$$

Numerical values for $\left\langle\sigma_{\text {res }}\right\rangle$ and $\left\langle\sigma_{\text {dis }}\right\rangle$ are given in Table 3. The efficiencies $\varepsilon_{i j}^{\mathrm{qel}}, \varepsilon_{i}^{\text {res }}$ and $\varepsilon_{i}^{\mathrm{dis}}$ should be estimated with the help of the MC simulation for QEL, RES and DIS samples separately; the factor $\Phi_{0} \sigma_{0} / N_{0}$ comes from the auxiliary process used for normalization.

Let us note that the smearing of the reconstructed neutrino energy is taken into account in (11) by the inverse matrix of QEL efficiencies.

Equation (11) can also be applied to the entire energy interval. In this case, we can use the usual notations for efficiencies as in (10). From the measured $\left\langle\sigma_{\text {qel }}\right\rangle$ we calculate the axial mass $M_{A}$ by using the Smith and Moniz formalism (see Fig. 4).
In the following subsections, we investigate the DIS and IMD processes which can both be used for the QEL crosssection normalization as just described.

Possible sources of systematic errors in our analysis procedure are discussed in Sect. 7.

\subsubsection{Selection of DIS events}

The phenomenology of neutrino DIS is well developed. Experimental data are in rather good agreement with theoretical predictions. The charged-current neutrino DIS is an inclusive process and for its selection from the data sample, the following criteria are enough.

- Fiducial volume cut. The primary vertex should be in the same FV as that defined for the QEL events, see (5).

- Muon identification and topology cut. At least two charged tracks should originate from the primary vertex; one of them should be identified as a muon $\left(\mu^{-}\right.$in the case of $v_{\mu} \mathrm{CC}$ and $\mu^{+}$for $\bar{v}_{\mu} \mathrm{CC}$ ).

- Background suppression. The third criterion is used to avoid contributions from the QEL and RES events. We have checked three different possibilities for it.

1. The total visible energy in the event should be $E_{v} \leqslant$ $300 \mathrm{GeV}$ and the reconstructed hadronic mass $W \geqslant$ $1.4 \mathrm{GeV}$; in this case the computation of $\left\langle\sigma_{\text {dis }}\right\rangle$ has been done for GRV98-LO PDF model according to the prescriptions in [65].

2. We keep the requirement for the reconstructed hadronic mass $(W \geqslant 1.4 \mathrm{GeV})$ but reduce the neutrino energy region to $40 \leqslant E_{v} \leqslant 200 \mathrm{GeV}$; theoretical calculation of $\left\langle\sigma_{\text {dis }}\right\rangle$ is also done with the help of [65].

3. Using the same neutrino energy interval as in $2(40 \leqslant$ $E_{v} \leqslant 200 \mathrm{GeV}$ ), we remove the cut on the reconstructed hadronic mass $W$. In this case, we take the total CC neutrino-nucleon cross section to be ${ }^{7}$

$$
\begin{aligned}
& \sigma_{\nu}^{\mathrm{tot}}\left(E_{\nu}\right) / E_{\nu}=(0.677 \pm 0.014) \times 10^{-38} \mathrm{~cm}^{2} / \mathrm{GeV}, \\
& \sigma_{\bar{\nu}}^{\text {tot }}\left(E_{\nu}\right) / E_{\nu}=(0.334 \pm 0.008) \times 10^{-38} \mathrm{~cm}^{2} / \mathrm{GeV}
\end{aligned}
$$

(PDG average [49]). The calculated $\left\langle\sigma_{\text {tot }}\right\rangle$ should be corrected due to the fact that NOMAD target is slightly non-isoscalar (0.984 for $v_{\mu}$ and 1.014 for $\left.\bar{v}_{\mu}\right)$.

The numerical results of the DIS events selection can be found in Table 5. For the QEL cross-section normalization we use results obtained with the last method (PDG based). Other two methods are also used in the analysis to control and minimize the overall systematic error on the final results. Thus, the final normalization is performed to the total

\footnotetext{
${ }^{7}$ The CHORUS measurement for the $\mathrm{CH}_{2}$ target [83] is consistent with this value.
} 
Table 5 Selection of the DIS events in $v_{\mu}$ and $\bar{v}_{\mu} C C$ samples. Total efficiency (in \%), expected purity of selected events (in \%), theoretical prediction for $\left\langle\sigma_{\mathrm{dis}}\right\rangle$, observed $N_{\text {dat }}$ and corrected $N_{0}$ number of events in experimental data are given for each variant of DIS selection described above

\begin{tabular}{|c|c|c|c|c|c|c|}
\hline \multirow[t]{2}{*}{ Variant of DIS selection } & \multicolumn{3}{|l|}{$v_{\mu}$ sample } & \multicolumn{3}{|c|}{$\bar{v}_{\mu}$ sample } \\
\hline & $\overline{1}$ & 2 & 3 & 1 & 2 & 3 \\
\hline Efficiency & 82.95 & 86.84 & 88.52 & 75.46 & 81.40 & 83.20 \\
\hline Purity & 97.10 & 98.62 & 99.62 & 71.48 & 72.57 & 73.95 \\
\hline$N_{\text {dat }}$, events & 676702.0 & 267517.0 & 276018.0 & 17744.0 & 7996.0 & 8500.0 \\
\hline$N_{0}$, events & 792162.0 & 303790.7 & 310617.3 & 16807.1 & 7128.6 & 7553.4 \\
\hline Relative flux $\Phi_{0}$ & 1 & 0.144 & 0.144 & 1 & 0.106 & 0.106 \\
\hline$\left\langle\sigma_{0}\right\rangle, 10^{-38} \mathrm{~cm}^{2}$ & 16.643 & 44.876 & 46.069 & 4.876 & 20.124 & 21.999 \\
\hline$C^{-1}, 10^{-43} \mathrm{~cm}^{2}$ & 2.101 & 2.127 & 2.136 & 29.012 & 29.924 & 30.872 \\
\hline
\end{tabular}

$v_{\mu}\left(\bar{v}_{\mu}\right)$ CC cross section. We also checked that this normalization is consistent with two previous calculations based on approach from [65] within $1.6 \%(5.9 \%)$ for $v_{\mu}\left(\bar{v}_{\mu}\right) \mathrm{CC}$ sample.

\subsubsection{Selection of inverse muon decay events}

Inverse muon decay $v_{\mu} e^{-} \rightarrow \mu^{-} v_{e}$ is a purely leptonic process, which is well known both on theoretical and experimental grounds. Its cross section in the Born approximation is

$\sigma_{\text {imd }}\left(E_{v}\right)=\sigma_{\text {as }} E_{v}\left(1-\frac{m_{\mu}^{2}}{2 m_{e} E_{v}}\right)^{2}$.

The numerical value of the constant $\sigma_{\text {as }}$ calculated in the framework of the Standard Model was found to be in good agreement with experimental measurements [84]:

$\sigma_{\mathrm{as}}=\frac{2 m_{e} G_{F}^{2}}{\pi}=1.723 \times 10^{-41} \mathrm{~cm}^{2} \mathrm{GeV}^{-1}$

The number of IMD events $N_{0}$ is proportional to its fluxaveraged cross section from (4):

$\left\langle\sigma_{\text {imd }}\right\rangle=1.017 \times 10^{-40} \mathrm{~cm}^{2}$

and expected to be at least 650 times smaller than the number of DIS events.

To select the IMD events we require:

- the primary vertex should be in the same fiducial volume as that used for identified QEL events, see (5);

- there is only one negatively charged track originating from the primary vertex; it should be identified as a muon;

- there are no veto chamber hits in the vicinity of the intersection point of the extrapolated muon track and the first drift chamber (quality cut, the same as for 1-track events from the QEL sample);

- the muon energy is above the threshold:

$$
E_{\mu} \geqslant \frac{m_{\mu}^{2}+m_{e}^{2}}{2 m_{e}}=10.93 \mathrm{GeV}
$$

- the transverse momentum $p_{\perp}$ of the muon produced in IMD event is very limited by kinematics: $p_{\perp}^{2} \leqslant 2 m_{e} E_{\mu}$.

In this sample the contamination from the reaction $\bar{v}_{e} e \rightarrow \mu^{-} \bar{v}_{\mu}$ is estimated to be at the level of $\sim 10^{-3}$, e.g. well below 1 event, since the ratio of the fluxes $\bar{v}_{e} / v_{\mu}$ is 0.0027 [30] while the ratio of the cross sections is $\sigma\left(\bar{v}_{e} e \rightarrow\right.$ $\left.\mu^{-} \bar{v}_{\mu}\right) / \sigma\left(v_{\mu} e \rightarrow \mu^{-} v_{e}\right) \approx 1 / 3$.

We determine the number of signal events $N_{\text {imd }}$ from the fit of the $p_{\perp}^{2}$ distribution to experimental data with the function $F\left(p_{\perp}^{2}\right)$ :

$F\left(p_{\perp}^{2}\right)=N_{\text {imd }} F_{\text {imd }}\left(p_{\perp}^{2}\right)+\left[N_{\text {dat }}-N_{\text {imd }}\right] F_{\text {bg }}\left(p_{\perp}^{2}\right)$,

where $F_{\text {imd }}$ and $F_{\text {bg }}$ are the normalized MC expectations for signal and background $p_{\perp}^{2}$ distributions; $N_{\text {dat }}$ denotes the number of events in real data which passed all selection criteria.

The QEL events are now playing the role of the most important background for the IMD selection. However, the contaminations from the RES and DIS events cannot be neglected since they distort the shape of the $p_{\perp}^{2}$ distribution. As usual, the relative contribution of each process to the expected background is proportional to the corresponding efficiency and flux-averaged cross section (see Table 3).

The expression (16) contains only one free parameter $N_{\text {imd }}$, which is the number of observed IMD events. Finally, for $p_{\perp}^{2}<0.03(\mathrm{GeV} / c)^{2}$ interval we find $N_{\text {imd }}=$ $436.0 \pm 28.5$ with the quality of the fit $\chi^{2} / N D F=0.89$ (see Fig. 13). Taking into account that the selection efficiency for the IMD events is $87.8 \%$ we report the total number of IMD events $N_{0}$, which can be used for the QEL normalization:

$N_{0}=496.6 \pm 32.5$.

The relative error for $\sigma_{0} / N_{0}$ in the IMD case is about $7 \%$ (due to the small statistics of the IMD sample). Nevertheless the normalization factor itself, $C^{-1}=2.048 \times 10^{-43} \mathrm{~cm}^{2}$, is in agreement (within $\sim 4 \%$ ) with the evaluation based on the DIS sample (see Table 5).

The use of the IMD process for the normalization is an interesting independent cross-check of the absence of problems in our procedure. In particular, it allows us to verify 


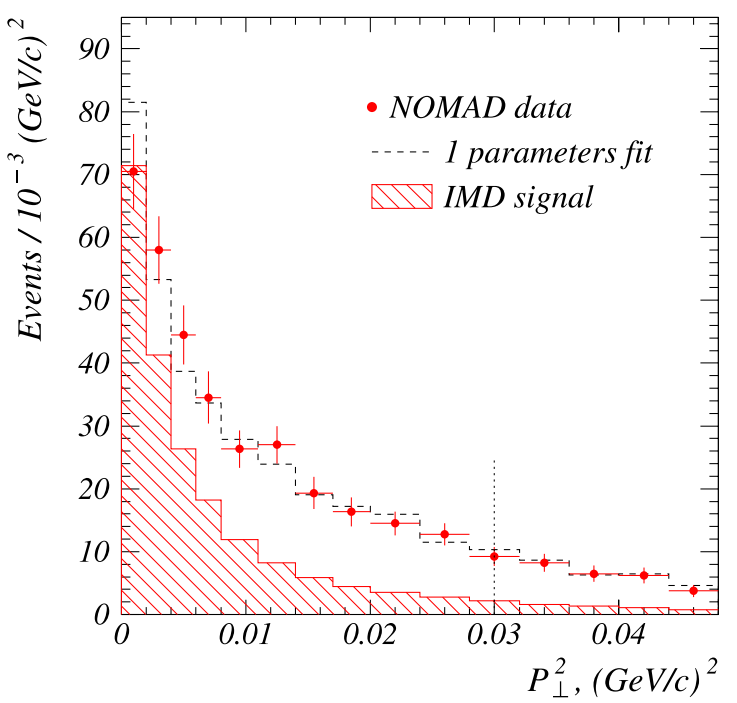

Fig. 13 Inverse Muon Decay: NOMAD experimental data, the $p_{\perp}^{2}$ distribution. Please note that the bins are of unequal widths: Bins 1 to 4 , 5 to 8 and 9 to last are respectively $0.001,0.0015$ and $0.002(\mathrm{GeV} / c)^{2}$ wide. Also note that the data above 0.03 were not used in the fit

that there are no effects arising from possible trigger inefficiencies in the selection of neutrino events consisting of a single muon going through the trigger planes.

\subsection{Axial mass measurement from the $Q^{2}$ distribution}

To extract the axial mass from the $Q^{2}$ distribution the experimental data are fitted to the theoretical predictions using a standard $\chi^{2}$ method. We bin the events in two variables $Q^{2}$ and $E_{v}$ (in the case of a single $E_{v}$ interval our procedure can be considered as the usual 1-dimensional fit). ${ }^{8}$

Let us enumerate bins with index $i=1 . . N_{B}$; bin $i=$ $N_{B}+1$ contains events which fall outside of the investigated $\left(E_{v}, Q^{2}\right)$ region. It is convenient to define boundaries in such a way that each bin with $i=1 . . N_{B}$ contains approximately the same number of experimental events passing all identification criteria.

A minimization functional is:

$\chi^{2}\left(M_{A}\right)=\sum_{i=1}^{N_{B}} \frac{\left[N_{i}^{\mathrm{dat}}-N_{i}^{\mathrm{th}}\left(M_{A}\right)\right]^{2}}{N_{i}^{\mathrm{th}}\left(M_{A}\right)}$

where $N_{i}^{\mathrm{dat}}$ is the number of events in the $i$ th bin of the experimental distribution, while $N_{i}^{\text {th }}$ is a superposition of the normalized MC background $N_{i}^{\mathrm{bg}}$ and the expected QEL sig-

\footnotetext{
${ }^{8}$ In practice it is convenient to use dimensionless variables $(a, b)$ instead of $\left(E_{v}, Q^{2}\right)$. Then, $E_{v}=E_{v}^{\min }+a\left(E_{v}^{\max }-E_{v}^{\min }\right)$ and $Q^{2}=$ $Q_{\min }^{2}\left(E_{v}\right)+b\left[Q_{\max }^{2}\left(E_{v}\right)-Q_{\min }^{2}\left(E_{v}\right)\right]$. So, $a, b \in[0,1]$.
}

nal:

$N_{i}^{\mathrm{th}}\left(M_{A}\right)=N_{i}^{\mathrm{bg}}+C \sum_{j=1}^{N_{B}+1} \varepsilon_{i j}^{\mathrm{qel}} \Phi_{j}\left\langle\tilde{\sigma}_{\mathrm{qel}}\right\rangle_{j}$.

This equation is similar to (8), $N_{i}^{\text {bg }}$ being defined in the same way as in (9); $\varepsilon_{i j}^{\text {qel }}$ is the probability that a QEL event simulated in the $j$ th bin is reconstructed in the $i$ th bin. The QEL scattering dynamics is described by the following term:

$$
\begin{aligned}
& \left\langle\tilde{\sigma}_{\mathrm{qel}}\right\rangle_{i}=\frac{1}{\Phi_{i}} \int_{\Omega_{i}} \frac{d \sigma}{d Q^{2}}\left(E, Q^{2}, M_{A}\right) \Phi(E) d E d Q^{2}, \\
& \left.\Phi_{i}\left\langle\tilde{\sigma}_{\mathrm{qel}}\right\rangle_{i}\right|_{i=N_{B}+1}=\left\langle\sigma_{\mathrm{qel}}\right\rangle-\sum_{j=1}^{N_{B}} \Phi_{j}\left\langle\tilde{\sigma}_{\mathrm{qel}}\right\rangle_{j} .
\end{aligned}
$$

Here $\Omega_{i}$ denotes the $\left(E_{v}, Q^{2}\right)$ interval, which corresponds to the $i$ th bin; $d \sigma / d Q^{2}$ is the differential QEL cross section on bound target nucleon (see formulae in [32]).

The coefficient $C$ can be defined in either of two ways:

1. the $N_{i}^{\text {th }}$ distribution is normalized to the total number of events in the experimental data:

$$
\sum_{i=1}^{N_{B}} N_{i}^{\mathrm{th}}=\sum_{i=1}^{N_{B}} N_{i}^{\mathrm{dat}} .
$$

In this case, the proposed method should be sensitive only to the shape of the distribution but not to the absolute number of identified events (contrary to the $M_{A}$ measurement from the total QEL cross section).

2. $C$ is defined in the same way as for the total QEL crosssection measurement, i.e. we use another process (DIS) for normalization:

$C=\frac{N_{0}}{\Phi_{0} \sigma_{0}}$.

If we sum over the $Q^{2}$ variable for the investigated $\left(E_{\nu}, Q^{2}\right)$ interval, finding the $M_{A}$ parameter from (18) becomes nothing else than the numerical resolution of (8). Therefore, this variant of the fit can be considered as a simultaneous fit of the total and differential cross sections; henceforth, we shall refer to it as $\sigma \otimes d \sigma / d Q^{2}$ fit.

Figure 14 presents a comparison of the reconstructed $Q^{2}$ distribution with our MC prediction. The expected background contamination is also shown.

We can now apply the proposed methods to experimental data and measure the QEL cross section and axial mass $M_{A}$. The numerical results are reported in Sect. 8, while the discussion of the corresponding uncertainties is presented in the next section. 


\section{Systematic uncertainties}

We have studied several sources of systematic uncertainties, which are important for the measurement of the total QEL cross section and axial mass parameter. They are listed below.

1. Identification of QEL events; we vary the selection criteria within reasonable limits $(\mathcal{L}>0 \pm 0.4$ for 2 -track sample and $\theta_{h} / \pi>0.35 \pm 0.03$ for 1 -track sample).

The final result is found to be practically insensitive to the exact positions of the muon azimuth $\varphi_{\mu}$ cut and additional requirements for the $P_{\perp}^{\mathrm{mis}}, \alpha$ and $\theta_{h}$ variables: e.g. in the $v_{\mu}$ analysis a more strict cut $0.1 \pi<\varphi_{\mu}<$ $0.9 \pi$ leads to $0.8 \%$ variation in the measured cross sec-

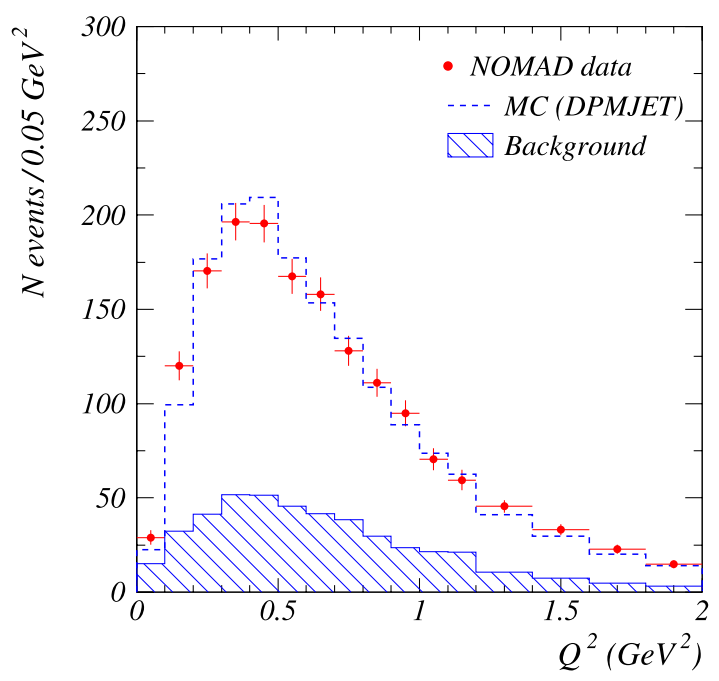

Fig. 14 The $Q^{2}$ distributions in identified QEL events tion while a change in the pre-cuts to $P_{\perp}^{\text {mis }}<0.9 \mathrm{GeV}$, $\alpha / \pi>0.75$ and $0.18 \pi<\theta_{h}$ leads to an uncertainty of $0.4 \%$.

2. Uncertainty in the total (mainly DIS) charged-current muon neutrino cross section, which enters both in the normalization factor $\sigma_{0} / N_{0}$ and in the subtraction of the corresponding DIS background (the experimental error on $\left\langle\sigma_{\text {dis }}\right\rangle$ is $2.1 \%$ for $v_{\mu} \mathrm{CC}$ and $2.4 \%$ for $\bar{v}_{\mu} \mathrm{CC}$ ).

3. Uncertainty in the RES cross section, which determines the contamination admixture of the single resonant pion events in the identified QEL sample (we assume 10\% error on $\left\langle\sigma_{\text {res }}\right\rangle$ both for neutrino and antineutrino cases, see e.g. [56]).

4. FSI interactions (we vary $\tau_{0}$ and $\alpha_{\text {mod }}^{F}$ DPMJET parameters for fixed $M_{A}^{\mathrm{mc}}=1.03 \mathrm{GeV}$ ).

5. Uncertainty in the neutrino flux shape (the relative errors for each $E_{v}$ bin were taken from [30]).

6. Uncertainty, concerning to the axial mass value $M_{A}^{\mathrm{mc}}$, used for MC production.

7. Neutral current admixture (we assume 5\% error for the corresponding cross section, which can be found in Table 3).

8. Charge misidentification of the primary lepton (reconstructed $v_{\mu} \mathrm{CC}$ event is classified as $\bar{v}_{\mu} \mathrm{CC}$ and vice versa).

9. Contamination from coherent pion production (see Sect. 4.4).

In Table 6 we present our numerical estimations for systematic uncertainties (in the case of $v_{\mu}$ scattering, systematic errors were calculated for the mixture of 1-track and 2-track subsamples). One can see that the most important contributions come from the QEL identification procedure and from

Table 6 The relative systematic uncertainties (in \%) of the QEL cross section $\left\langle\sigma_{\mathrm{qel}}\right\rangle$ and axial mass $M_{A}$, measured in $v_{\mu} n \rightarrow \mu^{-} p$ and $\bar{v}_{\mu} p \rightarrow$ $\mu^{+} n$ reactions

\begin{tabular}{|c|c|c|c|c|c|}
\hline Source & $\left\langle\sigma_{\mathrm{qel}}\right\rangle_{v_{\mu}}$ & $M_{A}$ from $\left\langle\sigma_{\mathrm{qel}}\right\rangle_{v_{\mu}}$ & $M_{A}$ from $d \sigma_{v} / d Q^{2}$ & $\left\langle\sigma_{\mathrm{qel}}\right\rangle_{\bar{v}_{\mu}}$ & $M_{A}$ from $\left\langle\sigma_{\mathrm{qel}}\right\rangle_{\bar{v}_{\mu}}$ \\
\hline \multicolumn{6}{|l|}{1 QEL identification procedure: } \\
\hline likelihood or $\theta_{h}$ cut & 3.5 & 3.2 & 2.4 & 4.3 & 4.2 \\
\hline$\varphi_{\mu}$ cut & 0.8 & 0.7 & 0.3 & - & - \\
\hline$P_{\perp}^{m i s}, \alpha$ and $\theta_{h}$ precuts & 0.4 & 0.4 & 0.4 & - & - \\
\hline $2 \delta\left(\left\langle\sigma_{d i s}\right\rangle\right), \delta\left(\sigma_{0}\right)$ & 2.9 & 2.6 & 0.2 & 4.2 & 4.2 \\
\hline $3 \delta\left(\left\langle\sigma_{r e s}\right\rangle\right)$ & 4.0 & 3.6 & 0.6 & 7.6 & 7.4 \\
\hline 4 Nuclear reinteractions & 1.8 & 1.6 & 6.5 & - & - \\
\hline 5 Shape of neutrino spectrum $(\delta(\Phi))$ & 1.6 & 1.5 & 0.1 & 5.9 & 5.6 \\
\hline $6 \delta\left(M_{A}^{m c}\right)$ & 1.0 & 0.9 & 0.9 & 1.4 & 1.4 \\
\hline $7 \mathrm{NC}$ contribution & $<0.1$ & $<0.1$ & - & 1.1 & 1.1 \\
\hline 8 Muon misidentification & $<0.1$ & $<0.1$ & - & 1.0 & 1.0 \\
\hline 9 Coherent pion production & $<0.1$ & $<0.1$ & $<0.1$ & 1.1 & 1.1 \\
\hline Total & 6.7 & 6.0 & 7.0 & 11.6 & 11.1 \\
\hline
\end{tabular}




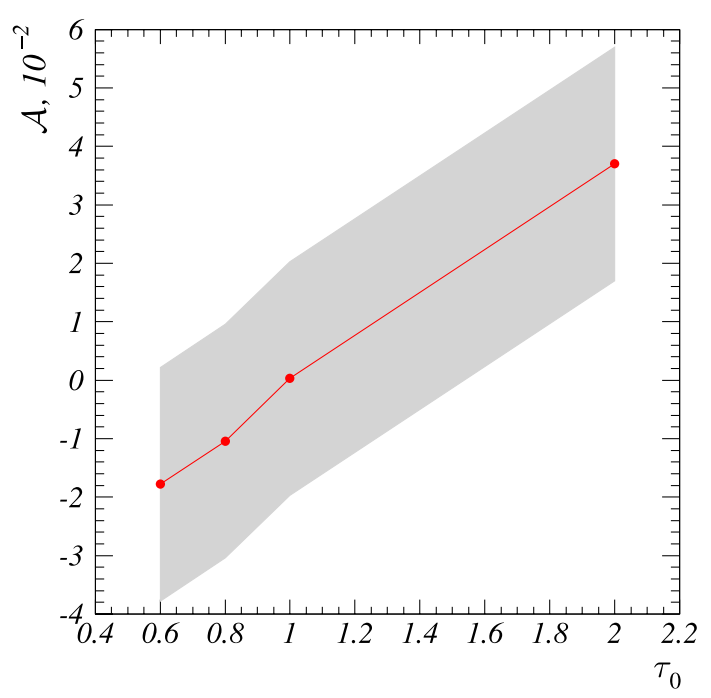

Fig. 15 The asymmetry $\mathcal{A}$ as a function of the formation time parameter $\tau_{0}$

the uncertainty on the non-QEL processes contribution to the selected sample of signal events.

The nuclear reinteractions (FSI effect) significantly affect the neutrino sample only (see Table 9), while in the antineutrino case the influence of the nuclear reinteractions is expected to be negligible.

In the case of $\bar{v}_{\mu}$ QEL scattering one measures only the $\mu^{+}$in the final state, while the outgoing neutron is not seen. This event topology can be changed only in the case of charged particle production through the intra-nuclear cascade. The fraction of those events is very small according to our MC estimations.

For $v_{\mu}$ scattering, the cross sections can be calculated separately for both the 1-track and 2-track subsamples of identified QEL events or for their mixture. We can then compare the results and choose whichever one has the minimal total error. In our case it was obtained for the combined 1-track and 2-track sample, which was found to be almost insensitive to the variation of DPMJET parameters (see Sect. 8 for explanations).

In order to estimate the error due to the FSI modeling we consider the following asymmetry ratio:

$\mathcal{A}=\frac{\sigma_{1}-\sigma_{2}}{2\left(\sigma_{1}+\sigma_{2}\right)}$,

where $\sigma_{1}$ and $\sigma_{2}$ denotes to the $v_{\mu}$ QEL cross sections calculated from the 1-track and 2-track subsamples respectively. The systematic errors in the measured $\sigma_{1}$ and $\sigma_{2}$ cross sections (here we take into account all possible sources of uncertainties, listed above, except FSI effects) provide an uncertainty in $\mathcal{A}$ which is found to be equal $\sim 0.02$. The dependence of the asymmetry ratio $\mathcal{A}$ on the $\tau_{0}$ parameter is shown in Fig. 15. One can conclude that allowed bounds for the formation time parameter are $\tau_{0}=1.0 \pm 0.5$. It was also found that the final results are not very sensitive to modifications of the second parameter of the DPMJET package $-\alpha_{\text {mod }}^{F}$. Using the default values $M_{A}^{\mathrm{mc}}=1.03 \mathrm{GeV}$, $\alpha_{\text {mod }}^{F}=0.6$ and varying $\tau_{0}$ within the interval $[0.5,1.5]$ one can obtain the systematic uncertainty for the measured QEL neutrino cross section and axial mass value due to the modeling of FSI effects.

The uncertainty on the shape of the (anti)neutrino spectrum is important for the measurement of $\sigma_{\mathrm{qel}}$ as a function of neutrino energy $E_{v}$. But it does not affect both the fluxaveraged cross section $\left\langle\sigma_{\mathrm{qel}}\right\rangle$ and the $M_{A}$ extraction from the $Q^{2}$ distribution.

The uncertainty due to the primary lepton misidentification and neutral currents comes into play through the subtraction of the corresponding background from the selected DIS sample, that is, from the normalization factor. The admixture of those events into the identified QEL events is negligible.

\section{Results}

\section{$8.1 v_{\mu} n \rightarrow \mu^{-} p$ sample}

The results of our analysis for the $v_{\mu}$ sample are summarized in Table 9. We measure the flux-averaged QEL cross section in the neutrino energy interval 3-100 GeV (see (11)) for the 1-track and 2-track samples as well as for their mixture (which is called Combined in Table 9). For each $\left\langle\sigma_{\mathrm{qel}}\right\rangle$ we calculate the corresponding axial mass value, $M_{A}$. Results on $M_{A}$ extraction both from the standard $Q^{2}$ fit and from the combined $\sigma \otimes d \sigma / d Q^{2}$ fit are also given. These measurements are repeated for several QEL MC with different values of input parameters (the axial mass $M_{A}$ was varied between 0.83 and $1.23 \mathrm{GeV}$ in steps of $0.1 \mathrm{GeV}$; the formation time $\tau_{0}$ was allowed to take a value of $0.6,1.0$ and 2.0; the correction factor $\alpha_{\text {mod }}^{F}$ was varied within the interval $[0.54,0.69])$. On top of this the NUANCE QEL MC with its own treatment of FSI effects is used for cross-checks.

We then observe that $M_{A}$ recalculated from the measured $\left\langle\sigma_{\mathrm{qel}}\right\rangle$ depends on $\tau_{0}$ if one refers to the 1-track or the 2-track samples. Specifically, the measured $M_{A}$ value increases with increasing $\tau_{0}$ when extracted from the 1-track sample while it decreases when extracted from the 2-track sample. This can be understood if we take into account the fact that the $\tau_{0}$ parameter controls the probability for an outgoing nucleon to be involved in an intranuclear cascade. Increasing $\tau_{0}$ then increases the fraction of QEL events with reconstructed proton and thus populates the 2-track sample to the detriment of the 1-track sample. This is the reason for the systematic overestimation of $M_{A}$ extracted from the 1-track sample alone and its underestimation when extracted from the 
Fig. 16 Comparison of our $\left\langle\sigma_{\mathrm{qel}}\right\rangle_{v_{\mu}}$ measurements as a function of the neutrino energy in the 1-track and 2-track subsamples (for the best parameter $\tau_{0}=1.0$ ) with the final $\left\langle\sigma_{\mathrm{qel}}\right\rangle_{v_{\mu}}$ values measured using the full event sample; see Table 7. An artificial shift is applied for the neutrino energy to make the overlapping points visible

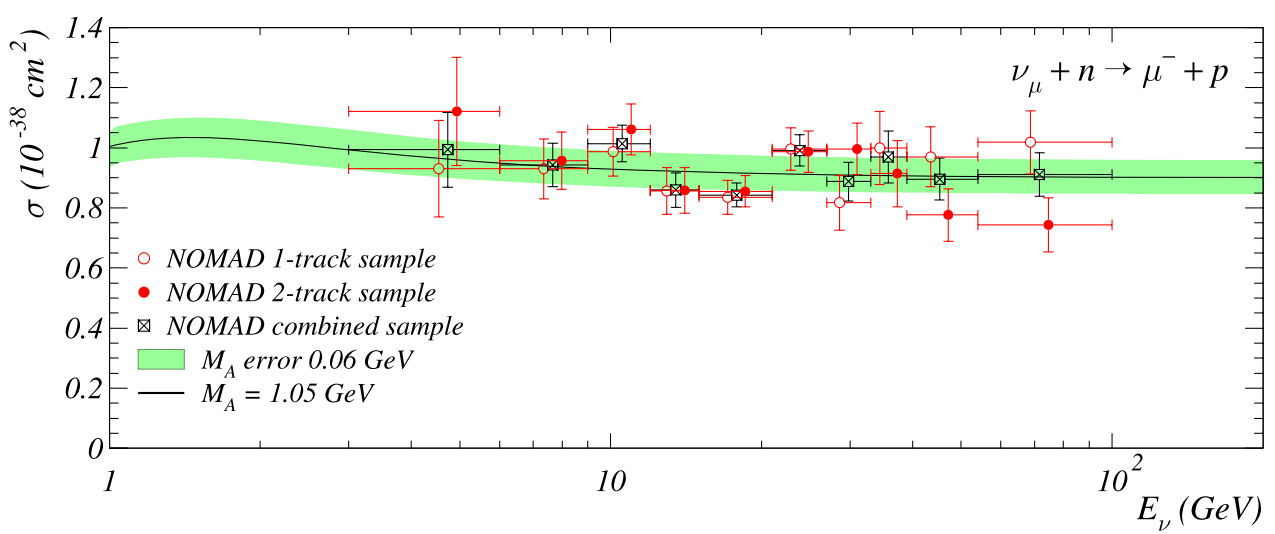

2-track sample alone. However the value of $M_{A}$ extracted from the combination of the 1-track and 2-track samples is almost insensitive to variations of the $\tau_{0}$ parameter.

We also find that using the QEL Monte Carlo with $\tau_{0}=1$ and $\alpha_{\text {mod }}^{F}=0.6$ provides the most accurate prediction for the ratio between the 1-track and 2-track samples (and hence the most adequate description of the FSI): in this case the flux-averaged QEL cross section stays approximately the same whether measured from the 1-track sample or from the 2-track sample (see Table 9). This allows us to exclude the MC sets with $\tau_{0}=0.6$ and 2.0 from further considerations.

Figure 16 shows a comparison of our $\left\langle\sigma_{\mathrm{qel}}\right\rangle_{v_{\mu}}$ measurements as a function of the neutrino energy in the 1-track and 2-track subsamples (for the best parameter $\tau_{0}=1.0$ ) with the final $\left\langle\sigma_{\mathrm{qel}}\right\rangle_{\nu_{\mu}}$ values measured using the full event sample.

Similarly we have observed that when using the full sample (1-track and 2-track) the measured $M_{A}$ is not very sensitive to modifications of the $\alpha_{\text {mod }}^{F}$ parameter. And using the NUANCE simulation code as a cross check gives a very consistent picture: the $M_{A}$ value extracted from the 1-track sample is also different from the one extracted from the 2-track sample, while the value obtained with the combined sample nicely agrees with our measurement with the best FSI parameters. Thus, our results for the neutrino case are

$\left\langle\sigma_{\mathrm{qel}}\right\rangle_{v_{\mu}}=(0.92 \pm 0.02$ (stat) \pm 0.06 (syst) $) \times 10^{-38} \mathrm{~cm}^{2}$,

$M_{A}=1.05 \pm 0.02$ (stat) \pm 0.06 (syst) GeV.

This result (25) is indeed in agreement with both the standard fit of the $Q^{2}$ distribution:

$M_{A}=1.07 \pm 0.06$ (stat) \pm 0.07 (syst) $\mathrm{GeV}$

and the fit of the combined $\sigma \otimes d \sigma / d Q^{2}$ distribution of the NOMAD data:

$M_{A}=1.06 \pm 0.02$ (stat) \pm 0.06 (syst) $\mathrm{GeV}$ (see Table 9, these results are obtained with a QEL MC using $M_{A}=1.03 \mathrm{GeV}$ ).

We use the 2-track sample only to extract $M_{A}$ from the fit of the $Q^{2}$ distribution since in this case the purity of QEL identification is rather high ( $\sim 74 \%$, see Table 4$)$. The results depend on the input MC parameters (axial mass and formation time) but still are in nice agreement with the results of the extraction of $M_{A}$ from the measured QEL cross section based also on a 2-track sample analysis. This can be considered as an additional confidence for our measurements using the full QEL sample.

The measured cross section of the $v_{\mu} n \rightarrow \mu^{-} p$ reaction as a function of the neutrino energy is presented in Table 7 and is shown in Figs. 17 and 18. These results are compared to the previous measurements performed with deuterium and heavy nuclei targets (see discussion in Sect. 2).

\section{$8.2 \bar{v}_{\mu} p \rightarrow \mu^{+} n$ sample}

In the $\bar{v}_{\mu}$ case the event topology is just a single $\mu^{+}$, thus the uncertainties in the treatment of FSI effect almost do not influence the event selection. Since our measurement of the cross section of the $\bar{v}_{\mu} p \rightarrow \mu^{+} n$ reaction is based on a 1-track sample only, we do not show the dependence of the results on the variation of the $\tau_{0}$ and $\alpha_{\text {mod }}^{F}$ parameters. Instead we display a dependence on the input $M_{A}$ in Table 10. The results for the measured $M_{A}$ are found to be quite stable. In Fig. 19 we show the measured $\bar{v}_{\mu} p \rightarrow \mu^{+} n$ cross section as a function of the antineutrino energy superimposed with the theoretical curve drawn with $M_{A}=1.06 \pm 0.14 \mathrm{GeV}$ and with nuclear effects according to the standard relativistic Fermi gas model. Table 8 summarizes our results for the $\bar{v}_{\mu} p \rightarrow \mu^{+} n$ cross-section measurement in the different antineutrino energy intervals. The cross sections are measured on a carbon target and also recalculated for a free nucleon. The statistical and systematic errors are both provided. The observed number of events in the data, the predicted number of background events, the background subtracted and efficiency corrected number of events are also shown. 
Table 7 Cross section of quasi-elastic neutrino scattering (in units of $10^{-38} \mathrm{~cm}^{2}$, statistical and systematic errors). $\sigma_{\text {carbon }}$ is measured for the NOMAD nuclear target and normalized per 1 neutron; $\sigma_{\text {Free }}=\sigma_{\text {Nucl }} / g$ is the cross section for the free target neutron (the factor $g$ is calculated according to the Smith-Moniz model; see [32]). The number of selected events in raw data $N_{\text {dat }}$, the estimated background contamination $N_{\text {bg }}$ and the number of events $N_{\text {cor }}$ corrected for background and efficiency are also reported. The difference in the total number of data events with respect to Table 4 (13683 versus 14021) is due to the additional cut on the neutrino energy $3<E_{v}(\mathrm{GeV})<100$

\begin{tabular}{lrrrrrr}
\hline$E_{v}$ & $\left\langle E_{v}\right\rangle$ & $N_{\text {dat }}$ & \multicolumn{1}{c}{$N_{\text {bg }}$} & $N_{\text {cor }}$ & $\left(\sigma \pm \delta \sigma_{\text {stat }} \pm \delta \sigma_{\text {syst }}\right)_{\text {Nucl }}$ & $\left(\sigma \pm \delta \sigma_{\text {stat }} \pm \delta \sigma_{\text {syst }}\right)_{\text {Free }}$ \\
\hline $3-6$ & 4.7 & 396 & 211.6 & 660.6 & 0.9940 .1250 .078 & 1.0570 .1330 .083 \\
$6-9$ & 7.7 & 1115 & 580.2 & 1663.4 & 0.9420 .0720 .078 & 1.0010 .0770 .083 \\
$9-12$ & 10.5 & 1683 & 835.3 & 2591.1 & 1.0140 .0610 .075 & 1.0770 .0650 .080 \\
$12-15$ & 13.5 & 1647 & 834.9 & 2310.7 & 0.8590 .0570 .070 & 0.9130 .0600 .075 \\
$15-21$ & 17.8 & 2815 & 1451.6 & 3766.8 & 0.8430 .0400 .067 & 0.8960 .0430 .071 \\
$21-27$ & 23.8 & 2040 & 956.2 & 3084.7 & 0.9910 .0520 .070 & 1.0530 .0550 .075 \\
$27-33$ & 29.8 & 1279 & 610.5 & 1816.8 & 0.8880 .0640 .073 & 0.9430 .0680 .077 \\
$33-39$ & 35.8 & 852 & 400.9 & 1246.2 & 0.9700 .0860 .078 & 1.0300 .0910 .083 \\
$39-54$ & 45.3 & 1008 & 496.1 & 1397.8 & 0.8960 .0700 .074 & 0.9510 .0740 .078 \\
$54-100$ & 71.7 & 848 & 416.9 & 1191.5 & 0.9110 .0730 .077 & 0.9670 .0780 .082 \\
$3-100$ & 23.4 & 13683 & 6794.2 & 19718.2 & 0.9190 .0170 .060 & 0.9760 .0180 .063 \\
\hline
\end{tabular}

Table 8 The same as Table 7 but for the antineutrino

\begin{tabular}{lrrrrll}
\hline$E_{v}$ & $\left\langle E_{v}\right\rangle$ & $N_{\text {dat }}$ & \multicolumn{1}{l}{$N_{\text {bg }}$} & \multicolumn{1}{l}{$N_{\text {cor }}$} & $\left(\sigma \pm \delta \sigma_{\text {stat }} \pm \delta \sigma_{\text {syst }}\right)_{\text {Nucl }}$ & $\left(\sigma \pm \delta \sigma_{\text {stat }} \pm \delta \sigma_{\text {syst }}\right)_{\text {Free }}$ \\
\hline $3-6$ & 4.5 & 291 & 176.4 & 181.9 & 0.5850 .0970 .079 & 0.6390 .1060 .086 \\
$6-9$ & 7.4 & 292 & 182.6 & 159.5 & 0.7100 .1250 .093 & 0.7670 .1350 .100 \\
$9-15$ & 11.8 & 464 & 286.2 & 254.1 & 0.8510 .1140 .096 & 0.9130 .1220 .103 \\
$15-24$ & 19.0 & 425 & 274.0 & 210.8 & 0.8220 .1250 .095 & 0.8780 .1340 .102 \\
$24-42$ & 31.5 & 411 & 252.5 & 223.0 & 1.0090 .1410 .098 & 1.0750 .1500 .104 \\
$42-100$ & 60.1 & 288 & 181.9 & 145.9 & 0.9930 .1730 .099 & 1.0560 .1840 .105 \\
$3-100$ & 19.7 & 2171 & 1353.6 & 1182.5 & 0.8110 .0530 .081 & 0.8660 .0560 .086 \\
\hline
\end{tabular}

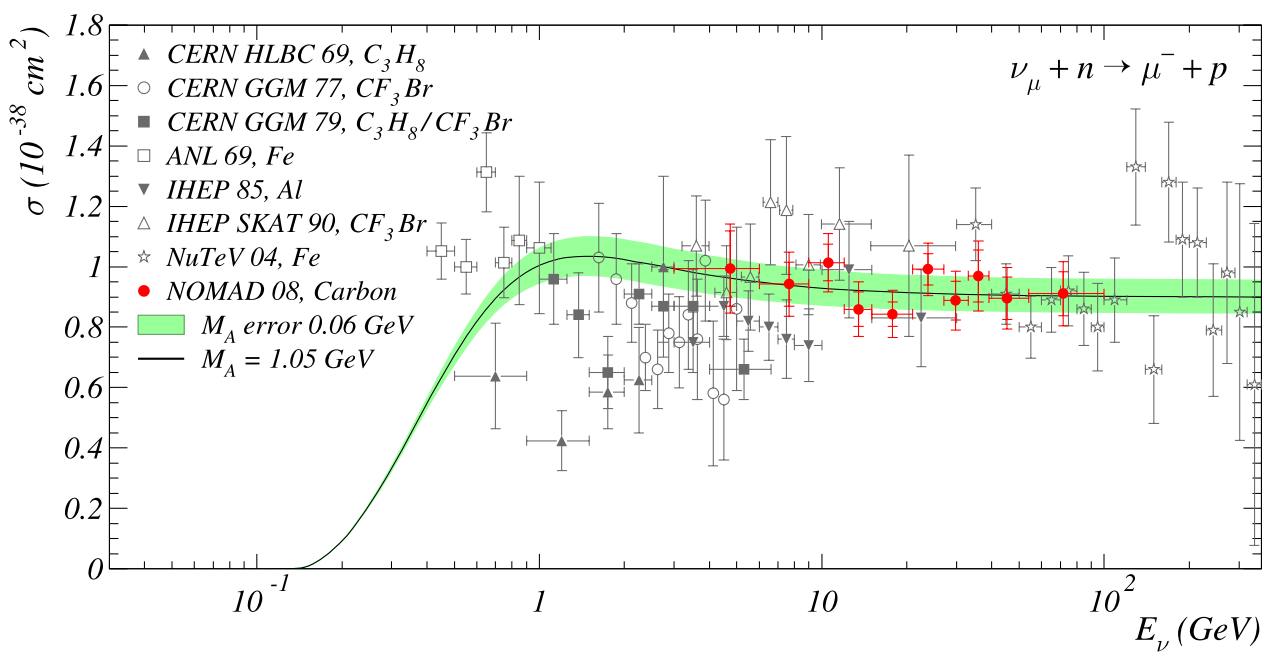

Fig. 17 Comparison of NOMAD $\left\langle\sigma_{\mathrm{qel}}\right\rangle_{v_{\mu}}$ measurements with previous experimental data on $v_{\mu}$ scattering off heavy nuclei (ANL 69 (Spark-chamber) [1], NuTeV 04 (FermiLab) [23], CERN HLBC 69 (CERN, Heavy Liquid Bubble Chamber) [14], CERN GGM 77 (CERN, Gargamelle BC) [15], CERN GGM 79 [17], IHEP 85 (IHEP, spark-chamber) [20], IHEP SCAT 90 (IHEP, BC) [22]). The solid line corresponds to the calculations according to the relativistic Fermi gas model by Smith and Moniz [72] for carbon (with binding energy $E_{b}=25.6 \mathrm{MeV}$ and Fermi momentum $P_{F}=221 \mathrm{MeV} / c$ ) using the $M_{A}$ value obtained in the NOMAD experiment. The error band takes into account both statistical and systematic uncertainties of the present analysis 


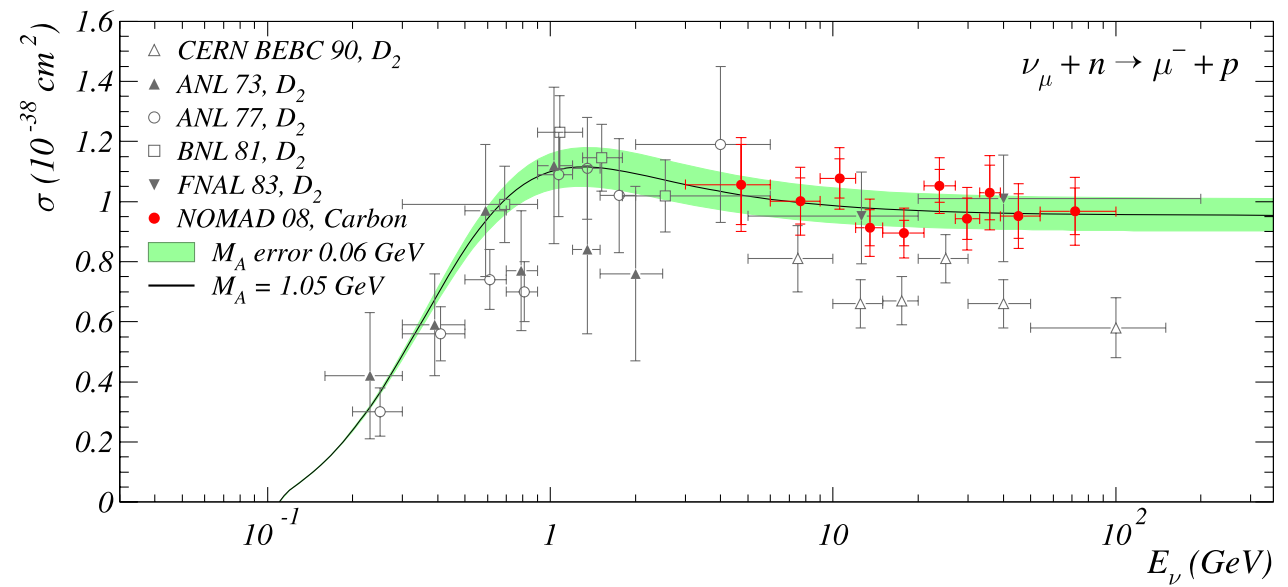

Fig. 18 Comparison of NOMAD $\left\langle\sigma_{\mathrm{qel}}\right\rangle_{v_{\mu}}$ measurements with previous $v_{\mu} D$ experimental data (ANL 73 (Argonne 12-foot BC) [2], ANL 77 [3], BNL 81 (Brookhaven 7-foot BC) [6], FNAL 83 (FermiLab 15-foot BC) [9], BEBC 90 (CERN, Big European Bubble Chamber) [18]; corrections for nuclear effects have been made by the authors of the experiments). NOMAD measurements were divided by a factor $g=\sigma_{\text {Nucl }} / \sigma_{\text {Free }}$ which was calculated according to the SmithMoniz model (see [32]). The solid line and error band corresponds to the $M_{A}$ value obtained in the NOMAD experiment
Table 9 Parameters of the QEL MC simulation (axial mass $M_{A}^{\mathrm{mc}}$ and parameters of FSI modeling) are listed in the first three columns. The intermediate columns contain results of the QEL $v_{\mu}$ cross-section measurement (in units of $10^{-38} \mathrm{~cm}^{2}$, without errors) for the different topology of identified events (with or without reconstructed proton track). The axial mass value obtained from the fit of $Q^{2}$ distribution and $\sigma \otimes d \sigma / d Q^{2}$ fit $\left(Q_{\mathrm{lim}}^{2}=0.2-4 \mathrm{GeV}^{2}\right)$ are given in the last columns of the table; only the statistical errors for the axial mass were shown. One can see that the final $M_{A}$ measurement for the combined sample has a very weak dependence on the initial $M_{A}^{\mathrm{mc}}$ used in the simulation (e.g. variation of $M_{A}^{\mathrm{mc}}$ in a rather large interval from 0.83 to 1.23 leads to a variation of the measured $M_{A}$ of about 0.03 )

\begin{tabular}{|c|c|c|c|c|c|c|c|c|c|c|c|c|}
\hline \multicolumn{3}{|c|}{ MC parameters } & \multicolumn{2}{|c|}{ Single track } & \multicolumn{2}{|c|}{ Two tracks } & \multicolumn{2}{|c|}{ Combined } & \multicolumn{2}{|c|}{ Fit of $Q^{2}$ distribution } & \multicolumn{2}{|c|}{ Fit of $\sigma \otimes d \sigma / d Q^{2}$} \\
\hline$\tau_{0}$ & $\alpha_{\text {mod }}^{F}$ & $M_{A}^{\mathrm{mc}}$ & $\sigma_{\mathrm{qel}}$ & $M_{A}$ & $\sigma_{\mathrm{qel}}$ & $M_{A}$ & $\sigma_{\mathrm{qel}}$ & $M_{A}$ & $\overline{M_{A} \pm \delta M_{A}}$ & $\chi^{2}$ & $\overline{M_{A} \pm \delta M_{A}}$ & $\chi^{2}$ \\
\hline 0.60 & 0.60 & 0.83 & 0.863 & 0.990 & 1.014 & 1.148 & 0.915 & 1.047 & 1.1130 .057 & 19.4 & 1.1050 .018 & 19.3 \\
\hline 1.00 & 0.60 & 0.83 & 0.885 & 1.015 & 0.956 & 1.090 & 0.912 & 1.043 & 1.0950 .057 & 11.6 & 1.0580 .018 & 11.9 \\
\hline 2.00 & 0.60 & 0.83 & 0.918 & 1.050 & 0.851 & 0.977 & 0.892 & 1.021 & 0.9600 .093 & 17.4 & 0.9650 .018 & 17.4 \\
\hline 0.60 & 0.60 & 0.93 & 0.882 & 1.011 & 1.015 & 1.148 & 0.928 & 1.061 & 1.1350 .056 & 10.0 & 1.1190 .018 & 10.0 \\
\hline 1.00 & 0.60 & 0.93 & 0.893 & 1.023 & 0.942 & 1.074 & 0.911 & 1.043 & 1.0750 .060 & 13.5 & 1.0650 .018 & 13.5 \\
\hline 2.00 & 0.60 & 0.93 & 0.931 & 1.063 & 0.844 & 0.968 & 0.896 & 1.026 & 1.0090 .069 & 12.2 & 0.9710 .018 & 12.4 \\
\hline 0.60 & 0.60 & 1.03 & 0.910 & 1.041 & 0.977 & 1.110 & 0.935 & 1.067 & 1.0160 .051 & 25.8 & 1.0940 .017 & 27.1 \\
\hline 1.00 & 0.60 & 1.03 & 0.919 & 1.051 & 0.918 & 1.050 & 0.919 & 1.051 & 1.0730 .059 & 18.7 & 1.0590 .018 & 18.7 \\
\hline 2.00 & 0.60 & 1.03 & 0.950 & 1.083 & 0.819 & 0.939 & 0.896 & 1.026 & 0.9930 .079 & 18.4 & 0.9680 .018 & 18.4 \\
\hline 0.60 & 0.60 & 1.13 & 0.946 & 1.079 & 0.979 & 1.113 & 0.959 & 1.092 & 1.0310 .077 & 24.9 & 1.1090 .017 & 26.4 \\
\hline 0.80 & 0.60 & 1.13 & 0.948 & 1.081 & 0.926 & 1.058 & 0.940 & 1.073 & 1.0920 .056 & 13.8 & 1.0790 .018 & 13.8 \\
\hline 1.00 & 0.60 & 1.13 & 0.962 & 1.096 & 0.904 & 1.035 & 0.940 & 1.072 & 1.1000 .062 & 19.4 & 1.0600 .018 & 19.6 \\
\hline 2.00 & 0.60 & 1.13 & 0.995 & 1.129 & 0.789 & 0.904 & 0.906 & 1.037 & 0.9990 .080 & 18.0 & 0.9560 .018 & 18.2 \\
\hline 0.60 & 0.60 & 1.23 & 0.994 & 1.127 & 0.925 & 1.058 & 0.967 & 1.100 & 1.0390 .053 & 20.7 & 1.0880 .018 & 21.2 \\
\hline 0.80 & 0.60 & 1.23 & 0.996 & 1.129 & 0.904 & 1.035 & 0.959 & 1.092 & 1.0130 .039 & 21.1 & 1.0660 .017 & 21.7 \\
\hline 1.00 & 0.60 & 1.23 & 1.000 & 1.134 & 0.879 & 1.008 & 0.951 & 1.085 & 0.9700 .087 & 20.1 & 1.0510 .017 & 21.3 \\
\hline 2.00 & 0.60 & 1.23 & 1.038 & 1.171 & 0.777 & 0.889 & 0.921 & 1.053 & 0.9960 .079 & 20.9 & 0.9640 .018 & 16.3 \\
\hline 0.80 & 0.54 & 1.03 & 0.921 & 1.053 & 0.963 & 1.097 & 0.937 & 1.070 & 1.1130 .054 & 20.9 & 1.0790 .018 & 21.1 \\
\hline 0.80 & 0.57 & 1.03 & 0.921 & 1.052 & 0.950 & 1.083 & 0.932 & 1.064 & 1.0720 .062 & 15.5 & 1.0670 .018 & 15.5 \\
\hline 0.80 & 0.60 & 1.03 & 0.920 & 1.051 & 0.959 & 1.092 & 0.935 & 1.067 & 1.0900 .064 & 12.7 & 1.0890 .018 & 12.7 \\
\hline 0.80 & 0.63 & 1.03 & 0.912 & 1.044 & 0.953 & 1.087 & 0.928 & 1.060 & 1.0820 .062 & 15.8 & 1.0840 .018 & 15.7 \\
\hline 0.80 & 0.66 & 1.03 & 0.905 & 1.035 & 0.933 & 1.066 & 0.916 & 1.047 & 0.9890 .091 & 19.9 & 1.0670 .017 & 20.9 \\
\hline 0.80 & 0.69 & 1.03 & 0.904 & 1.035 & 0.940 & 1.072 & 0.918 & 1.049 & 0.9370 .113 & 15.7 & 1.0700 .017 & 18.0 \\
\hline
\end{tabular}




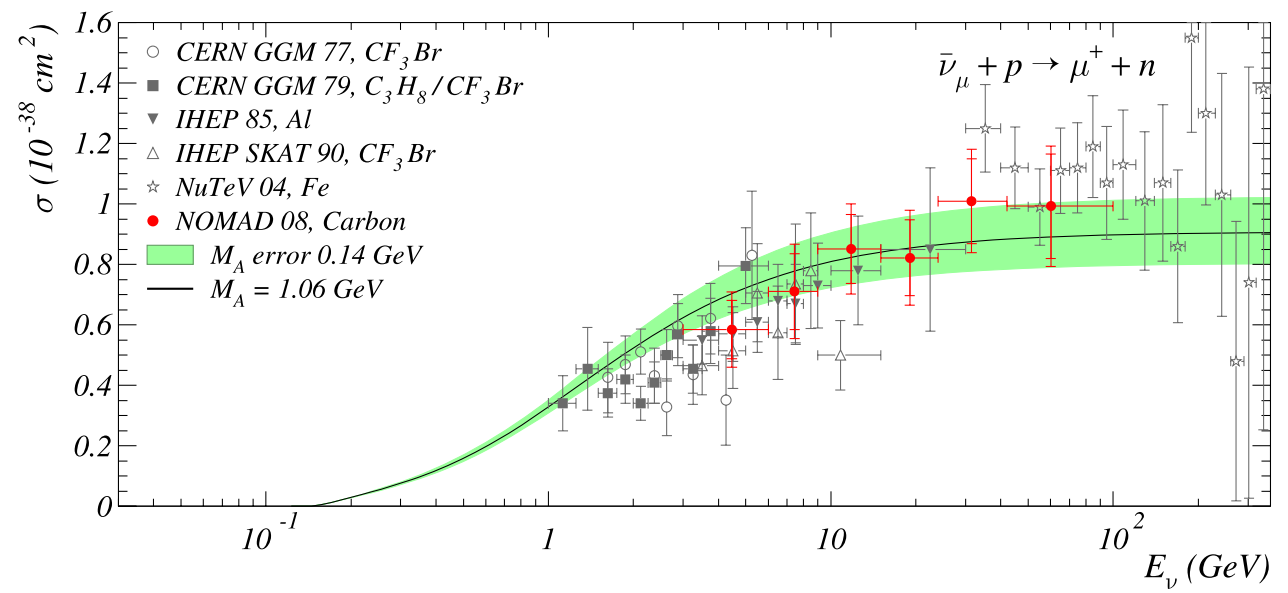

Fig. 19 Comparison of NOMAD $\left\langle\sigma_{\mathrm{qel}}\right\rangle_{\bar{v}_{\mu}}$ measurements, Table 8, with previous experimental data on $\bar{v}_{\mu}$ scattering off heavy nuclei (CERN GGM 77 [15], CERN GGM 79 [16], IHEP 85 [20], IHEP SCAT 90 [22] and NuTeV 04 [23]). The solid line corresponds to

Table 10 The results of QEL $\bar{v}_{\mu}$ cross-section measurement. The parameters of the DPMJET model are $\tau_{0}=1.0, \alpha_{\text {mod }}^{F}=0.6$

\begin{tabular}{lll}
\hline$M_{A}^{\mathrm{mc}}$ & $\sigma_{\mathrm{qel}}$ & $M_{A}$ \\
\hline 0.83 & 0.794 & 1.042 \\
0.93 & 0.799 & 1.048 \\
1.03 & 0.811 & 1.063 \\
1.13 & 0.834 & 1.094 \\
1.23 & 0.861 & 1.127 \\
\hline
\end{tabular}

Our final results for the antineutrino case are

$$
\begin{aligned}
& \left\langle\sigma_{\mathrm{qel}}\right\rangle_{\bar{v}_{\mu}}=(0.81 \pm 0.05 \text { (stat) } \pm 0.09 \text { (syst) }) \times 10^{-38} \mathrm{~cm}^{2} \\
& M_{A}=1.06 \pm 0.07 \text { (stat) } \pm 0.12 \text { (syst) } \mathrm{GeV}
\end{aligned}
$$

\section{Conclusions}

The cross-section measurement of the $\nu_{\mu} n \rightarrow \mu^{-} p$ and $\bar{v}_{\mu} p \rightarrow \mu^{+} n$ reactions on nuclear target was performed and reported in this article. The samples used in the analysis consist of 14,021 neutrino and 2,237 antineutrino events, which were identified as quasi-elastic neutrino scattering among the experimental data collected by the NOMAD Collaboration.

We have discussed in details the analysis procedure and the most significant sources of systematic error. Special attention was paid to the influence of the FSI effects on the measured physical quantities. The DPMJET code was used to simulate these FSI effects. We also proposed a method for tuning the intranuclear cascade parameters (mainly the formation time $\tau_{0}$ ), which was then used to reduce the corresponding systematic uncertainty. the calculations according to the relativistic Fermi gas model by Smith and Moniz using the $M_{A}$ value obtained in the NOMAD experiment. The error band takes into account both statistical and systematic uncertainties of the present analysis

For the $v_{\mu}$ case stable results have been obtained with the combined 1-track and 2-track samples since they are almost insensitive to the FSI effects.

The results for the flux-averaged QEL cross sections in the (anti)neutrino energy interval 3-100 GeV are

$\left\langle\sigma_{\mathrm{qel}}\right\rangle_{v_{\mu}}=(0.92 \pm 0.02($ stat $) \pm 0.06($ syst $)) \times 10^{-38} \mathrm{~cm}^{2}$

and

$\left\langle\sigma_{\mathrm{qel}}\right\rangle_{\bar{v}_{\mu}}=(0.81 \pm 0.05($ stat $) \pm 0.09($ syst $)) \times 10^{-38} \mathrm{~cm}^{2}$

for neutrino and antineutrino, respectively.

The axial mass $M_{A}$ was calculated from the measured cross sections: we find $M_{A}=1.05 \pm 0.06 \mathrm{GeV}$ from the $v_{\mu}$ sample and $M_{A}=1.06 \pm 0.14 \mathrm{GeV}$ from the $\bar{v}_{\mu}$ sample. The $M_{A}$ parameter was also extracted from the fit of the $Q^{2}$ distribution in the high purity sample of $v_{\mu}$ quasi-elastic 2-track events (with a reconstructed proton track). It was found to be consistent with the values calculated from the cross sections.

Our results are in agreement with the existing world average value $[33,36]$ and do not support the results found in recent measurements from the $\mathrm{NuTeV}$ [23], K2K [24, 25] and MiniBooNE [26] Collaborations, which reported somewhat larger values, however still compatible with our results within their large errors. A summary of existing experimental data on the axial mass measurements in neutrino and antineutrino experiments is shown in Fig. 20.

It should also be noted that the preliminary results reported earlier by the NOMAD Collaboration for the 2-track sample only $[85,86]$ suffered from a large systematic bias related to an improper treatment of the FSI effects in the simulation program. They should be now superseded by the new measurements reported here. 


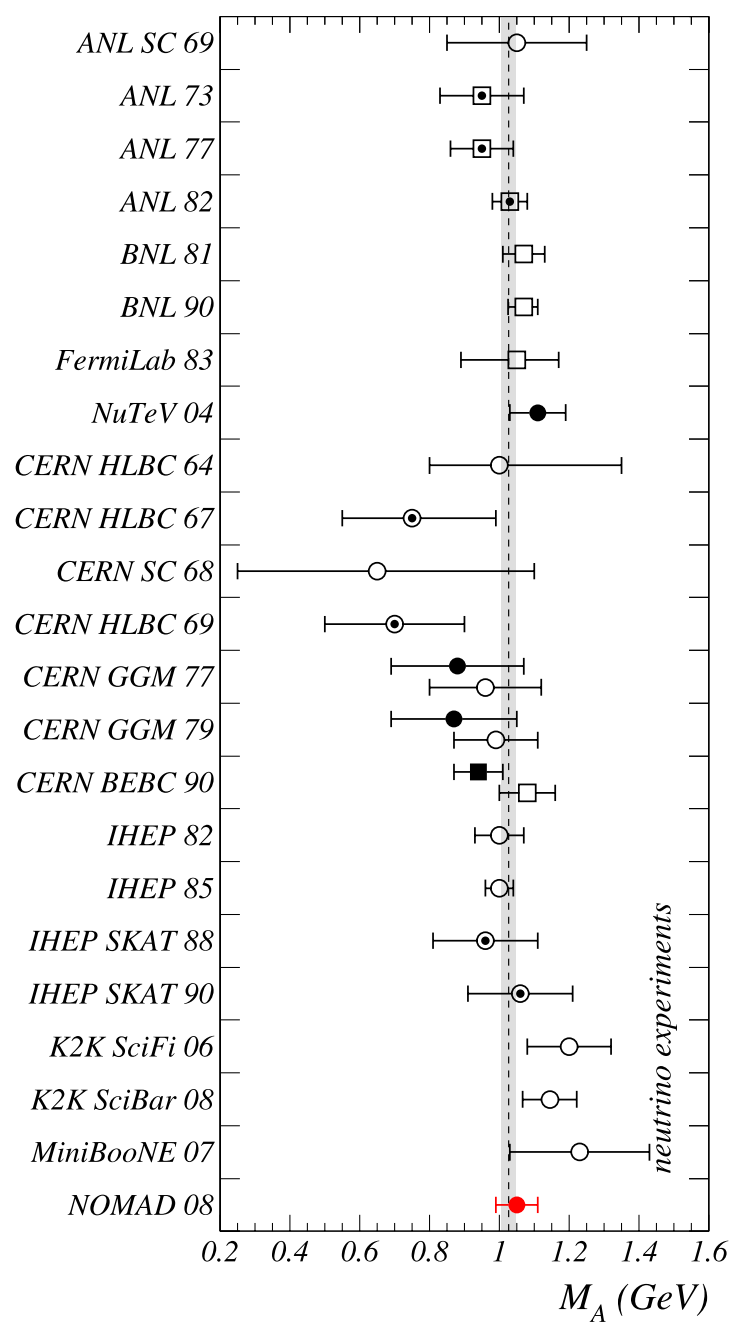

Fig. 20 A summary of existing experimental data: the axial mass $M_{A}$ as measured in neutrino (left) and antineutrino (right) experiments. Points show results obtained both from deuterium filled BC

Acknowledgements The experiment was supported by the following agencies: Australian Research Council (ARC) and Department of Industry, Science, and Resources (DISR), Australia; Institut National de Physique Nucléaire et Physique des Particules (IN2P3), Commissariat à l'Energie Atomique (CEA), Ministère de l'Education Nationale, de l'Enseignement Supérieur et de la Recherche, France; Bundesministerium für Bildung und Forschung (BMBF), Germany; Istituto Nazionale di Fisica Nucleare (INFN), Italy; Institute for Nuclear Research of the Russian Academy of Sciences, Russia; Joint Institute for Nuclear Research; Russian Foundation for Basic Research (grant 08-02-00018); Fonds National Suisse de la Recherche Scientifique, Switzerland; Department of Energy, National Science Foundation, the Sloan and the Cottrell Foundations, USA.

We thank the management and staff of CERN and of all participating institutes for their vigorous support of the experiment. Particular thanks are due to the CERN SPS accelerator and beam-line staff for the magnificent performance of the neutrino beam. Special thanks for useful discussions of theoretical issues go to K. Kuzmin and V. Naumov; we are grateful to prof. J. Ranft for the important explanations and technical assistance with the DPMJET code. V. Lyubushkin is very
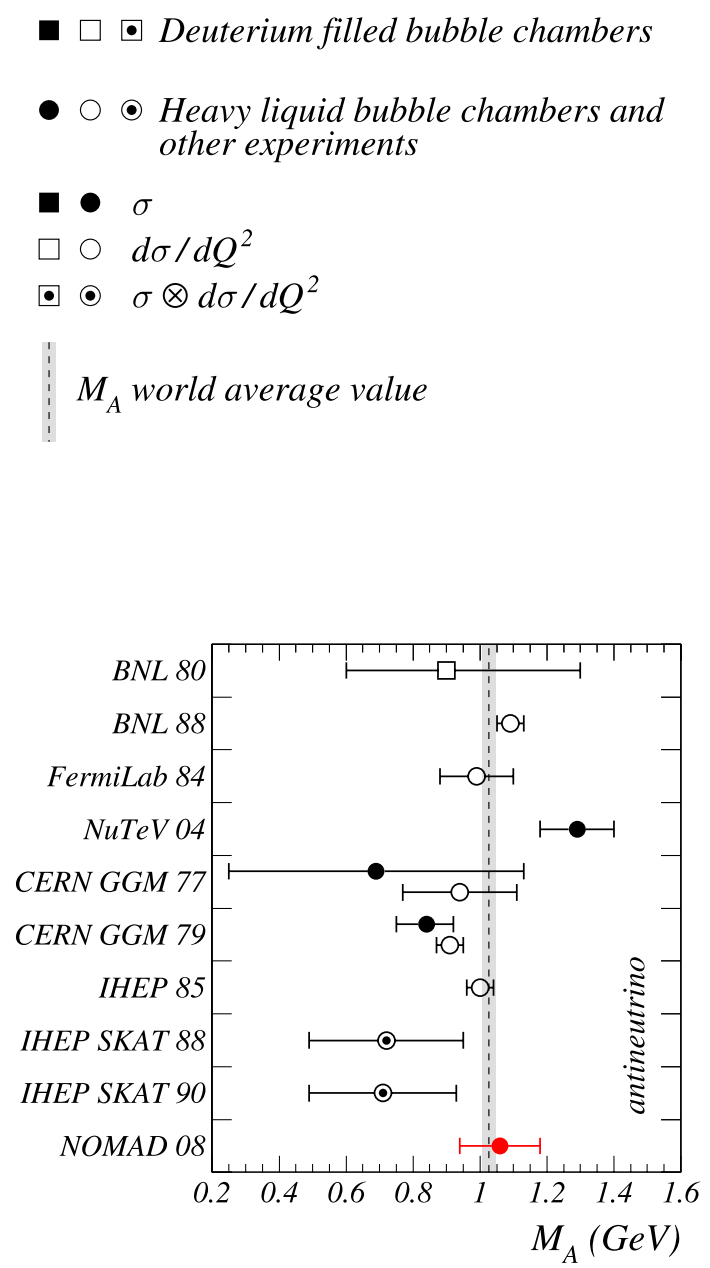

(squares) and from heavy liquid BC and other experiments (circles). The dashed line corresponds to the so-called world average value $M_{A}=1.026 \pm 0.021 \mathrm{GeV}$ (see the review in [33])

grateful to the LPNHE (Paris) for the warm hospitality and financial support during the final stage of this work.

\section{References}

1. R.L. Kustom, D.E. Lundquist, T.B. Novey, A. Yokosawa, F. Chilton, Phys. Rev. Lett. 22, 1014 (1969)

2. W.A. Mann et al., Phys. Rev. Lett. 31, 844 (1973)

3. S.J. Barish et al., Phys. Rev. D 16, 3103 (1977)

4. K.L. Miller et al., Phys. Rev. D 26, 537 (1982)

5. G. Fanourakis et al., Phys. Rev. D 21, 562 (1980)

6. N.J. Baker et al., Phys. Rev. D 23, 2499 (1981)

7. L.A. Ahrens et al., Phys. Lett. B 202, 284 (1988)

8. T. Kitagaki et al., Phys. Rev. D 42, 1331 (1990)

9. T. Kitagaki et al., Phys. Rev. D 28, 436 (1983)

10. A.E. Asratian et al., Phys. Lett. B 137, 122 (1984)

11. M.M. Block et al., Phys. Lett. 12, 281 (1964)

12. A. Orkin-Lecourtois et al., Nuovo Cimento A 50, 927 (1967)

13. M. Holder et al., Nuovo Cimento A 57, 338 (1968)

14. I. Budagov et al., Lett. Nuovo Cimento 2, 689 (1969) 
15. S. Bonetti et al., Nuovo Cimento A 38, 260 (1977)

16. N. Armenise et al., Nucl. Phys. B 152, 365 (1979)

17. M. Pohl et al., Lett. Nuovo Cimento 26, 332 (1979)

18. D. Allasia et al., Nucl. Phys. B 343, 285 (1990)

19. S.V. Belikov et al., Yad. Fiz. 35, 59 (1982)

20. S.V. Belikov et al., Z. Phys. A 320, 625 (1985)

21. H.J. Grabosch et al., Sov. J. Nucl. Phys. 47, 1032 (1988)

22. J. Brunner et al., Z. Phys. C 45, 551 (1990)

23. N. Suwonjandee, Ph.D. Thesis, University of Cincinnati (2004)

24. R. Gran et al., Phys. Rev. D 74, 052002 (2006). arXiv:hepex/0603034 (K2K Collaboration)

25. C. Mariani, AIP Conf. Proc. 981, 247 (2008) (K2K Collaboration)

26. A.A. Aguilar-Arevalo et al., Phys. Rev. Lett. 100, 032301 (2008). arXiv:0706.0926 [hep-ex] (MiniBooNE Collaboration)

27. A.A. Aguilar-Arevalo et al., arXiv:hep-ex/0601022 (SciBooNE Collaboration) (2006)

28. D. Drakoulakos et al., arXiv:hep-ex/0405002 (Minerva Collaboration) (2004)

29. J. Altegoer et al., Nucl. Instrum. Meth. A 404, 96 (1998) (NOMAD Collaboration)

30. P. Astier et al., Nucl. Instrum. Meth. A 515, 800 (2003). arXiv: hep-ex/0306022 (NOMAD Collaboration)

31. V.V. Lyubushkin, Ph.D. Thesis, JINR, Dubna (2008)

32. K.S. Kuzmin, V.V. Lyubushkin, V.A. Naumov, Eur. Phys. J. C 54, 517 (2008). arXiv:0712.4384 [hep-ph]

33. V. Bernard, L. Elouadrhiri, U.G. Meissner, J. Phys. G 28, R1 (2002). arXiv:hep-ph/0107088

34. H. Budd, A. Bodek, J. Arrington, arXiv:hep-ex/0308005 (2003)

35. H. Budd, A. Bodek, J. Arrington, Nucl. Phys. Proc. Suppl. 139, 90 (2005). arXiv:hep-ex/0410055

36. A. Bodek, S. Avvakumov, R. Bradford, H. Budd, Eur. Phys. J. C 53, 349 (2008). arXiv:0708.1946 [hep-ex]

37. M. Anfreville et al., Nucl. Instrum. Meth. A 481, 339 (2002). arXiv:hep-ex/0104012

38. G. Bassompierre et al., Nucl. Instrum. Meth. A 403, 363 (1998)

39. G. Bassompierre et al., Nucl. Instrum. Meth. A 411, 63 (1998)

40. J. Altegoer et al., Nucl. Instrum. Meth. A 428, 299 (1999) (NOMAD Collaboration)

41. D. Autiero et al., Nucl. Instrum. Meth. A 373, 358 (1996)

42. D. Autiero et al., Nucl. Instrum. Meth. A 411, 285 (1998)

43. P. Astier et al., Nucl. Phys. B 611, 3 (2001). arXiv:hep-ex/0106102 (NOMAD Collaboration)

44. P. Astier et al., Phys. Lett. B 570, 19 (2003). arXiv:hep-ex/ 0306037 (NOMAD Collaboration)

45. C.H. Llewellyn Smith, Phys. Rep. 3, 261 (1972)

46. M.F. Gari, W. Kruempelmann, Phys. Lett. B 274, 159 (1992)

47. E.L. Lomon, Phys. Rev. C 66, 045501 (2002). arXiv:nucl-th/ 0203081

48. E.L. Lomon, arXiv:nucl-th/0609020 (2006)

49. C. Amsler et al., Phys. Lett. B 667, 1 (2008). (Particle Data Group Collaboration)

50. D. Rein, L.M. Sehgal, Ann. Phys. 133, 79 (1981)

51. D. Rein, Z. Phys. C 35, 43 (1987)

52. K.S. Kuzmin, V.V. Lyubushkin, V.A. Naumov, Mod. Phys. Lett. A 19, 2815 (2004). arXiv:hep-ph/0312107
53. K.S. Kuzmin, V.V. Lyubushkin, V.A. Naumov, Nucl. Phys. Proc. Suppl. 139, 158 (2005). arXiv:hep-ph/0408106

54. A. Rodriguez et al., Phys. Rev. D 78, 032003 (2008). arXiv: 0805.0186 [hep-ex] (K2K Collaboration)

55. R.P. Feynman, M. Kislinger, F. Ravndal, Phys. Rev. D 3, 2706 (1971)

56. K. Furuno et al., Prepared for 2nd International Workshop on Neutrino-Nucleus Interactions in the Few GeV Region (NUINT 02), Irvine, California, 12-15 Dec. 2002 (2003)

57. G. Ingelman, A. Edin, J. Rathsman, Comput. Phys. Commun. 101, 108 (1997). arXiv:hep-ph/9605286

58. J.M. Levy, arXiv:hep-ph/0407371 (2004)

59. J.R. Ellis, A. Kotzinian, D.V. Naumov, Eur. Phys. J. C 25, 603 (2002). arXiv:hep-ph/0204206

60. T. Sjostrand, arXiv:hep-ph/9508391 (1995)

61. T. Sjostrand, Comput. Phys. Commun. 39, 347 (1986)

62. T. Sjostrand, M. Bengtsson, Comput. Phys. Commun. 43, 367 (1987)

63. M. Gluck, E. Reya, A. Vogt, Eur. Phys. J. C 5, 461 (1998). arXiv: hep-ph/9806404

64. C. Andreopoulos, Acta Phys. Polon. B 37, 2349 (2006) (GENIE Collaboration)

65. K.S. Kuzmin, V.V. Lyubushkin, V.A. Naumov, arXiv:hep-ph/ 0511308 (2005)

66. K.S. Kuzmin, V.V. Lyubushkin, V.A. Naumov, Phys. At. Nucl. 69, 1857 (2006)

67. L.J. Winton, Ph.D. Thesis, University of Melbourne (1999)

68. D. Rein, L.M. Sehgal, Nucl. Phys. B 223, 29 (1983)

69. D. Rein, Nucl. Phys. B 278, 61 (1986)

70. K. Hiraide et al., arXiv:0811.0369 [hep-ex] (SciBooNE Collaboration) (2008)

71. O. Benhar, A. Fabrocini, S. Fantoni, I. Sick, Nucl. Phys. A 579, 493 (1994)

72. R.A. Smith, E.J. Moniz, Nucl. Phys. B 43, 605 (1972)

73. S.A. Kulagin, R. Petti, Nucl. Phys. A 765, 126 (2006). arXiv: hep-ph/0412425

74. G. Battistoni, P. Lipari, J. Ranft, E. Scapparone, arXiv:hep-ph/ 9801426 (1998)

75. J. Ranft, Z. Phys. C 43, 439 (1989)

76. A. Ferrari, P.R. Sala, J. Ranft, S. Roesler, Z. Phys. C 70, 413 (1996). arXiv:nucl-th/9509039

77. D. Casper, Nucl. Phys. Proc. Suppl. 112, 161 (2002). arXiv:hep$\mathrm{ph} / 0208030$

78. Q. Wu et al., Phys. Lett. B 660, 19 (2008). arXiv:0711.1183 (NOMAD Collaboration)

79. E.A. Paschos, J.Y. Yu, M. Sakuda, Phys. Rev. D 69, 014013 (2004). arXiv:hep-ph/0308130

80. A. Marchionni, M. Veltri, NOMAD internal memo 98-023 (1998)

81. B.D. Yabsley, NOMAD internal memo 97-028 (1997)

82. B.D. Yabsley, NOMAD internal memo 98-011 (1998)

83. A. Kayis-Topaksu et al., Eur. Phys. J. C 30, 159 (2003) (CHORUS Collaboration)

84. P. Vilain et al., Phys. Lett. B 364, 121 (1995)

85. R. Petti, arXiv:hep-ex/0411032 (NOMAD Collaboration) (2004)

86. V.V. Lyubushkin, B.A. Popov, Phys. At. Nucl. 69, 1876 (2006) 